\title{
CARACTERÍSTICAS FRACTAIS DOS CONTORNOS DE BACIAS HIDROGRÁFICAS E DOS PERÍMETROS A ELES ASSOCIADOS: UMA ANÁLISE INVERSA RELATIVA ÀS IMPLICAÇÕES DIMENSIONAIS SOBRE OS DESCRITORES MORFOMÉTRICOS DE COMPACIDADE
}

\author{
FRACTAL CHARACTERISTICS OF CONTOURS OF HIDROGRAPHIC \\ BASINS AND PERIMETERS ASSOCIATED: AN INVERSE ANALYSIS \\ RELATED WITH THE DIMENSIONAL IMPLICATIONS ON \\ MORPHOMETRIC DESCRIPTORS OF COMPACTNESS
}

\author{
Luiz Eduardo Teixeira Ferreira \\ Centro Universitário das Faculdades Associadas de Ensino \\ Largo Engenheiro Paulo de Almeida Sandeville, 15. São João da Boa Vista, São Paulo, CEP: 13870-377, Brasil \\ Email: luizeduardotferreira@gmail.com
}

Lídia Maria Vala Ferreira

Teixeira \& Ferreira Engenharia Especializada Rua Carlos Eduardo Nacif, 173, Águas da Prata, São Paulo, CEP: 13890-000, Brasil

Email:ferreiradi@hotmail.com

\section{Informações sobre o Artigo}

Data de Recebimento:

07/03/2015

Data de Aprovação:

$04 / 07 / 2015$

Palavras-chave:

Descritores Morfométricos; Análise Fractal; Morfometria de Bacias.

\section{Keywords:}

Morphometric Descriptors; Fractal Analysis; Morphometry of Basins.

\section{Resumo:}

Neste artigo, uma análise dos mecanismos de obtenção dos descritores morfométricos é inicialmente procedida. Posteriormente, considerações relativas às características fractais dos contornos das bacias e dos perímetros a eles associados, são levadas a efeito. Por incorporarem grandezas geométricas em suas definições, fica evidenciada a necessidade de uma reavaliação relativa, tanto à qualidade, como à efetividade dos descritores morfométricos, tendo em vista que os mesmos são habitualmente computados por meio de ferramentas da geometria euclidiana. Para tanto, a técnica denominada Técnica da Caminhada sobre o Contorno, é adotada para a determinação das dimensões fractais, Df, dos contornos das bacias hidrográficas. Subsequentemente, essa técnica é explorada de maneira a obterem-se as dimensões fractais dos contornos de treze bacias e seis sub-bacias hidrográficas de diferentes características. Finalmente, dentro de procedimentos de análise inversa, as informações assim obtidas são utilizadas com vistas à determinação de contornos regularizados para essas bacias hidrográficas e de valores dos perímetros a eles associados, objetivando a reavaliação dos descritores de compacidade. Ao final, conclui-se que o modelo adotado conduz a reduções drásticas no número de pontos que constituem os novos contornos, com modificações insignificantes nas magnitudes das áreas ou descaracterizações das formas primitivas. Verificaram-se, ainda, reduções significativas nos Índices 
de Compacidade, $\mathrm{kC}$, e consequentes aumentos das Razões de Circularidade, $\mathrm{RC}$, parâmetros fundamentais para o desenvolvimento de análises morfométricas associadas às bacias hidrográficas, especialmente em atividades de avaliação de riscos de natureza hidrológica.

\begin{abstract}
:
In this paper, an analysis of the mechanisms for obtaining morphometric descriptors is initially proceeded. Subsequently, the fractal characteristics regarding the contours of the basins and its associated perimeters are carried out. By incorporating geometrical parameters in their definitions, the need for a reassessment on both, the quality, as well as the effectiveness of the morphometric descriptors, given that they are usually computed by Euclidean geometry tools, becomes evident. Therefore, the technique termed Divers Method or Waking Technique is adopted to determine the fractal dimension, Df, of the basin boundaries. Subsequently, this technique is exploited in order to obtain the fractal dimensions of the boundaries of thirteen basins and six sub-basins of different characteristics. Finally, within inverse analysis procedures, the information thus obtained is used in order to determine regularized boundaries for these watersheds and values of the perimeters associated with them, aiming the revaluation of compactness descriptors. Finally, it is concluded that the model adopted leads to drastic reductions in the number of boundary points that makes up the new contours, with insignificant changes in the magnitudes of the areas or distortion of primitive shapes. There were also significant reductions in Compactness Coefficient, $\mathrm{kC}$, and consequent increases in Circularity Ratio, RC, basic parameters used in the development of quantitative morphometric analyzes associated with watershed, especially in risk assessment of hydrological nature.
\end{abstract}

\section{Introdução}

Importantes indicadores utilizados em disciplinas que, de alguma maneira, trabalham com as características do relevo, diversos descritores morfométricos, a exemplo do Índice de Compacidade, $\mathrm{k}_{\mathrm{C}}$, definido por Gravelius (1914, apud JULCA, 2014) há exatamente um século, ou da Razão de Circularidade, $\mathrm{R}_{\mathrm{C}}$, proposta por Miller (1953), são parâmetros largamente utilizados em engenharia para a análise das bacias hidrográficas, no que diz respeito aos seus potenciais de geração de enchentes e picos de cheia.

Fundamentalmente, esses parâmetros estabelecem relações entre os perímetros associados aos contornos das bacias hidrográficas e as áreas por eles encerradas, de maneira a possibilitar a mensuração das características de circularidade e, consequentemente, de compacidade dessas bacias.

Sabe-se, já há algum tempo, que a determinação das grandezas geométricas citadas é altamente influenciada pela escala utilizada para a sua observação e mensuração.

Particularmente, a avaliação dos perímetros associados aos contornos das bacias hidrográficas ou a contornos de outras formas complexas, parece ainda constituir-se em atividade cercada por incertezas. Dentre essas incertezas cita-se a definição do nível de aprofundamento das observações em aplicações práticas, adequado ou necessário à quantificação das grandezas referidas, uma vez que a análise euclidiana das propriedades geométricas das formas ainda parece de difícil substituição, ao menos em curto prazo, no cotidiano das pessoas.

Por outro lado, os conceitos afetos às dimensões fractais consolidados por Mandelbrot (1977), assim como as metodologias deles decorrentes (e.g. RICHARDSON, 1961; MANDELBROT, 1967; SARKER e CHAUDHURI, 1994; DEEPA e TESSAMMA, 2010), são utilizados com o objetivo de determinarem-se as dimensões fractais das bacias hidrográficas, relativamente aos seus perímetros e às suas áreas.

Entende-se por fractalidade a propriedade de invariância por transformação de escala, em partes ou no todo de sua extensão (e com pelo menos uma dimensão fractal), apresentada por uma forma, objeto ou estrutura.

Em sua concepção original de estruturas e formas fractais, Mandelbrot (1977) deu foco também às noções de autossimilaridade e de autoafinidade.

Por autossimilaridade entende-se a propriedade apresentada por uma forma ou objeto a qual garante que toda e qualquer parte do mesmo retrate o todo desse objeto ou forma (no sentido de completude), e viceversa. Os fractais autoafins são aqueles que possuem 
dimensões fractais anisotrópicas, isto é, a sua dimensão fractal depende da direção na qual o escalonamento é procedido.

Como referido, simultaneamente ao conceito de autossimilaridade, Mandelbrot postulou a dimensão fractal como um invariante, ou seja, quando medida para uma forma ou objeto por meio da utilização de qualquer escala que fosse, esse escalar seria sempre o mesmo.

Portanto, o pressuposto sugerido por Mandelbrot de invariância desse escalar (que é um tensor de ordem zero), mediante uma transformação linear de simples escalonamento, é matematicamente necessário para que a dimensão fractal determinada em um dado procedimento esteja, de fato, correta.

Um exemplo clássico de autossimilaridade, é a curva ou floco de neve de von Koch, ilustrada na Figura 1 , que resulta de sucessivos desenvolvimentos dos lados que constituem a forma, segundo os padrões da forma inicial. Dessa maneira, a cada repetição do padrão, o perímetro da forma cresce, dando origem a " $n$ " novos desenvolvimentos da forma inicial, de tal maneira que a autossimilaridade fica evidenciada.

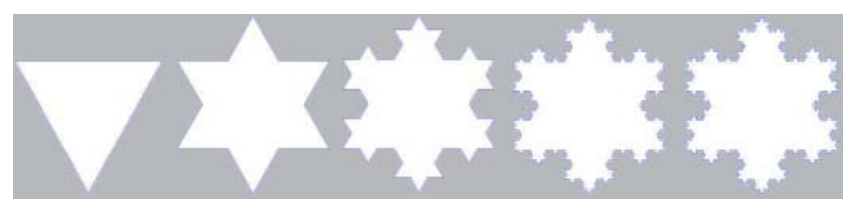

Figura 1 - Curva ou floco de neve de von Koch, após quatro repetições do algoritmo.

Por consequência, o contorno de uma forma puramente fractal não deve ser entendido como um desenvolvimento caótico, no sentido dado por Lorenz, (1995) qual seja, de um contorno randomicamente desenvolvido, de tal maneira que o resultado desse desenvolvimento conduza, de fato, a uma forma aleatória. Ao contrário, a partir dos conceitos de autossimilaridade e de autoafinidade conclui-se que as formas genuinamente fractais são extremamente organizadas e que os seus desenvolvimentos (ou crescimentos) ocorrem de maneira matematicamente previsível.

É sabido que as formas fractais, via de regra, apresentam dimensões intermediárias (não inteiras), portanto extrapolando "estritamente" as dimensões topológicas de Hausdorff-Besicovitch (FALCONER, 1990), conforme postulado por Mandelbrot.
Em outras palavras, no caso euclidiano unidimensional de uma curva, a sua dimensão fractal passa a apresentar valores superiores à unidade na medida em que o "grau" de complexidade espacial da mesma aumente. Esse conceito é aplicável, portanto aos contornos das formas e em especial ao tema aqui tratado, que são os contornos das bacias hidrográficas.

Igualmente, passam a apresentar dimensões superiores a dois para superfícies, na medida em que a complexidade espacial da forma aumente (eventualmente em direção a um volume) e superiores a 3, no caso de formas volumétricas. Entretanto, é um equívoco conceitual o entendimento de que todo fractal deva possuir dimensão não inteira. Com efeito, há fractais que possuem dimensões idênticas à dimensão euclidiana da sua projeção.

Todavia, tornou-se evidente que, com a variação da escala utilizada para a observação de formas irregulares e quantificação de grandezas geométricas a elas afetas (a exemplo dos perímetros, associados aos contornos e das áreas, associadas às superfícies), as magnitudes dessas grandezas passam a assumir valores diferentes daqueles determinados por meio da utilização da formulação clássica da geometria euclidiana.

Em outras palavras, essas grandezas, quando avaliadas da maneira tradicional, são efetivamente dependentes de escala, o que se aplica, consequentemente, às outras grandezas físicas delas decorrentes ou dependentes. Exemplos clássicos são fartamente encontrados na literatura relacionada à Mecânica da Fratura, a exemplo do cálculo da Energia de Fraturamento, $\mathrm{G}_{\mathrm{F}}$. (viz. BAZĂNT e PFEIFFER, 1987; BAZǍNT e KAZEMI, 1990; RILEM, 1990).

A grandeza referida representa a quantidade de energia a ser necessariamente dissipada para que o avanço de uma fissura, dita unitária, se verifique. Nas análises bidimensionais, a dissipação energética necessária ao avanço da fissura associa-se à extensão usualmente tortuosa da mesma e a uma largura unitária. Em análises tridimensionais, a associação da dissipação energética relaciona-se com as áreas das faces da fissura.

Ao serem examinadas por meio de técnicas mais sofisticadas, as topografias irregulares desses contornos e dessas superfícies revelam a existência de vales e picos que conduzem a perímetros e áreas globais de maiores magnitudes. Ao se amplificarem ainda mais o nível das observações, esses vales e cumes passam a evidenciar 
a existência de subvales e subcumes que conduzirão a valores ainda maiores e assim, sucessivamente.

Entretanto, essas novas formas e subformas não são legitimamente fractais, pois a recursividade ou repetitividade de um padrão primitivo não se verifica. Por outro lado, as superfícies de uma fissura podem possuir certas características aproximadamente fractais (apenas). Esse entendimento decorre da inexistência clara de fractalidade na geração dessas superfícies (UNDERWOOD e BANERJI,1986; PANDE et al., 1987; LUNG e MU, 1988 e HUANG et al., 1990 apud CONSTANTIN e IORDACHE, 2012).

Com base em situações físicas reais como a anteriormente discutida, SCHEIDEGGER (1970), HAKANSON (1978) e GOODCHILD (1980), apud SHELBERG et al. (1982) atenuaram o pré-requisito ou pressuposto de existência de auto similaridade à análise fractal. Nesse mesmo sentido, GOODCHILD (1980) pondera que a autossimilaridade é apenas um aspecto da abordagem fractal e que seria imprudente rejeitar todo o seu conceito, exclusivamente pela sua ausência, justificando, assim a aplicabilidade da análise fractal às diversas situações de aparente complexidade, desorganização ou aleatoriedade.

No entendimento dos autores deste artigo, a abordagem fractal é apenas um dentre os inúmeros enfoques disponíveis para a análise de fenômenos e processos que apresentam crescimentos complexos ou quase exponenciais. Em última análise, essa antiga ferramenta é aqui utilizada para o tratamento de novos problemas, como os ora discutidos.

\subsection{Implicações do Efeito de Escala Sobre ds Descritores Morfométricos}

Ao incorporarem grandezas geométricas em suas definições (perímetros e áreas), fica evidenciada a necessidade de uma reavaliação relativa, tanto à qualidade, como à efetividade dos descritores morfométricos, tendo em vista que os mesmos são habitualmente computados por meio de ferramentas da geometria euclidiana. Decorre desse fato que, não raramente, os indicadores assim obtidos passam a não refletir de maneira consistente (e confiável) as características das bacias hidrográficas, muitas vezes motivando perplexidades aos analistas.

Neste artigo, uma análise dos mecanismos de obtenção dos descritores morfométricos é inicialmente procedida. Posteriormente, considerações relativas às características fractais dos contornos e dos perímetros a eles associados são levadas a efeito. Não obstante existirem diversas técnicas para esse fim (KLINKEMBERG, 1994), a técnica introduzida por Richardson (1961) e posteriormente desenvolvida por Mandelbrot (1967), denominada Técnica da Caminhada sobre o Contorno, é adotada para a determinação das dimensões fractais, $\mathrm{D}_{\mathrm{f}}$, dos contornos das bacias hidrográficas

Subsequentemente, essa técnica é explorada de maneira a obterem-se as dimensões fractais dos contornos de treze bacias e seis sub-bacias hidrográficas de diferentes características, selecionadas para o desenvolvimento dos estudos.

Finalmente, dentro de procedimentos de análise inversa, as informações assim obtidas são utilizadas com vistas à definição de valores regularizados dos perímetros das bacias hidrográficas, objetivando a reavaliação dos descritores de compacidade.

\section{Considerações Sobre as Formas Regulares da Geometria Euclidiana e Descritores de Compacidade}

Quando analisadas por meio das ferramentas da geometria euclidiana, de maneira geral, as formas das figuras regulares apresentam relações claras entre o perímetro $\mathrm{P}$, associado ao contorno da forma, e a área A por ele encerrada. Para o caso bidimensional, podese escrever que:

$$
A=C_{2}\left(\frac{P}{C_{1}}\right)^{(d+1) / d}=C_{2}\left(\frac{P}{C_{1}}\right)^{D_{t}}
$$

onde $\mathrm{C}_{1}$ é uma constante relacionada ao caso unidimensional e $\mathrm{C}_{2}$ uma constante associada ao caso bidimensional. A variável $\mathrm{D}_{\mathrm{t}}$ é a dimensão topológica $\mathrm{e}$ $d$, assim como $\mathrm{D}_{\mathrm{t}}$ devem ser, obrigatoriamente, números inteiros. Para o caso em discussão, o valor de $d$ é a unidade, de tal maneira que a dimensão topológica da área A será igual a 2. Assim, a equação anterior pode ser reescrita da maneira que segue:

$$
A=\left(\frac{C_{2}}{C_{1}^{2}}\right)(P)^{2}
$$

Fazendo $\mathrm{C}_{2} / \mathrm{C}_{1}{ }^{2}=k$, que é a constante euclidiana de proporcionalidade entre a área e o perímetro, tem-se que:

$$
P=k \cdot \sqrt{A}
$$




\section{Características Fractais dos Contornos de Bacias Hidrográficas e dos Perímetros a eles Associados}

Para uma circunferência, por exemplo, o perímetro é dado por $\mathrm{P}=\pi \mathrm{D}$ e a área por $\mathrm{A}=\pi \mathrm{D}^{2} / 4$. Isolando-se $\mathrm{D}$ na equação do perímetro e substituindo-o na equação da área, obtém-se:

$$
A=\frac{\pi}{4}\left(\frac{P}{\pi}\right)^{2}=\frac{1}{4 \pi}(P)^{2}
$$

resultando que:

$$
P=k \cdot \sqrt{A}=2 \sqrt{\pi} \sqrt{A}
$$

\section{1 Índice de Compacidade, $\mathrm{K}_{\mathrm{c}}$, $\mathrm{e}$ Razão de Circularidade, $\mathbf{R}_{\mathrm{c}}$}

Esse descritor fundamenta-se na relação existente entre o perímetro da bacia hidrográfica em análise, $\mathrm{P}_{\mathrm{BH}}, \mathrm{e}$ o perímetro de uma circunferência imaginária que encerra área idêntica à da bacia hidrográfica, $\mathrm{A}_{\mathrm{BH}}$ (VILELLA e MATTOS, 1975; LANCASTRE e FRANCO, 1984).

Da manipulação direta da Equação 5, de maneira a estabelecer-se a razão entre o perímetro $\mathrm{P}$ e a área $\mathrm{A}$ da circunferência, entretanto, normalizando essa razão relativamente à constante $k$ de tal maneira que o seu valor mínimo seja unitário, obtém-se o Índice de Gravelius ou Índice de Compacidade, $\mathrm{k}_{\mathrm{C}}$ :

$$
\frac{P}{\sqrt{A}}=k=2 \sqrt{\pi}
$$

Fazendo-se $\mathrm{A}=\mathrm{A}_{\mathrm{BH}}$ e $\mathrm{P}=\mathrm{P}_{\mathrm{BH}}$, obtém-se desse conceito e da Equação 6 que:

$k_{C}=\frac{P}{k \sqrt{A}}=\frac{P}{2 \sqrt{\pi} \sqrt{A}}=\frac{\sqrt{\pi}}{2 \pi} \frac{P}{\sqrt{A}} \approx 0.2821 \frac{P}{\sqrt{A}}$

A Razão de Circularidade, $\mathrm{R}_{\mathrm{c}}$, introduzida por Miller (1953) é definida como sendo a razão entre a área da bacia hidrográfica, $\mathrm{A}_{\mathrm{BH}}$, e a área de uma circunferência com perímetro idêntico ao da bacia hidrográfica, $\mathrm{P}_{\mathrm{BH}}$. Matematicamente, a Razão de Circularidade pode ser obtida por meio da reorganização direta da Equação 4, $\operatorname{com} \mathrm{A}=\mathrm{A}_{\mathrm{BH}}$ e $\mathrm{P}=\mathrm{P}_{\mathrm{BH}}$ :

$$
R_{C}=\frac{4 \pi A}{P^{2}}
$$

Naturalmente, as relações dadas pelas Equações 7 e 8 poderiam ser obtidas a partir de manipulações algébricas das expressões decorrentes das definições de $\mathrm{k}_{\mathrm{C}}$ e $\mathrm{R}_{\mathrm{C}}$. Essas equações levam a inferir que, dentro de um enfoque meramente Euclidiano, os valores de $\mathrm{k}_{\mathrm{C}}$ e $\mathrm{R}_{\mathrm{C}}$, são necessariamente relacionáveis da maneira que segue:

$$
R_{C}=\frac{1}{k_{C}^{2}}
$$

De interesse às análises posteriormente realizadas neste trabalho, por hora satisfaz o entendimento de que quanto mais afastado de $2 \sqrt{ } \pi(\approx 3.5449)$ estiver a constante de proporcionalidade ${ }^{1} k$ apresentada na Equação 3, menor será a circularidade da bacia hidrográfica e, por consequência, menor será a sua compacidade.

\section{Formas não Euclidianas: Considerações Relativas aos Contornos Fractais.}

Diferentemente do caso das formas genuinamente euclidianas, para as formas fractais as equações anteriores via de regra não se verificam, isto é, o expoente que integra o segundo membro da Equação 1, por exemplo, não será, na maioria das vezes, um número inteiro.

No caso do enfoque fractal, a relação área-perímetro é dada pela Equação 10 (MANDERBROT, 1967):

$$
P^{\frac{2}{D_{f}}} \propto A
$$

a qual pode ser rescrita como:

$$
P^{\frac{1}{D_{f}}} \propto A^{\frac{1}{2}} \quad \Rightarrow \quad P \propto \sqrt{A^{D_{f}}}
$$

Chamando $\mathrm{k}^{*}$ à constante de proporcionalidade existente entre a área e o perímetro, a Equação 11 pode ser apresentada da maneira que segue (LOVEJOY, 1982):

$$
P=k^{*} \cdot \sqrt{A^{D_{f}}}
$$

\footnotetext{
${ }^{1}$ Para uma forma quadrada, por exemplo, ter-se-ia que $\mathrm{P}=4 \mathrm{~b}, \mathrm{~A}=\mathrm{b}^{2}$ e $k=4$, onde $\mathrm{b}$ é o valor do lado do quadrado. Para o triângulo equilátero, o valor de $\mathrm{k}$ é igual a $2\left(3^{3 / 4}\right)$. Para as elipses e deduzida a partir da Equação de Ramanujan (1962), essa constante é dada por: $k=\sqrt{\frac{\pi}{a b}}[3(a+b)-\sqrt{(3 a+b)(a+3 b)}]$ equação que se aplica igualmente à circunferência, caso elíptico particular onde se tem os semieixos a=b.
} 
onde $\mathrm{D}_{\mathrm{f}}$ é a dimensão fractal do contorno da forma.

Observe que essa equação aplica-se universalmente às formas regulares da geometria euclidiana, uma vez que, nesse caso $D_{f}=D_{t}=1$, que é dimensão topológica do perímetro e do contorno a ele associado. Decorre da Equação 12 que:

$$
k^{*}=\frac{P}{\sqrt{A^{D_{f}}}}
$$

\section{Determinação da Dimensão Fractal, $D_{f}$, Referente ao Perímetro Associado ao Contorno da Bacia Hi- drográfica.}

Como referido, diversas são as técnicas existentes para a determinação da dimensão fractal de formas lineares (KLINKEMBERG, 1994). Com o objetivo de estudar os contornos das bacias hidrográficas, os perímetros a eles associados e as suas dimensões fractais, $\mathrm{D}_{\mathrm{f}}$, adota-se como ponto de partida o método introduzido por Richardson (1961) e posteriormente desenvolvido por Mandelbrot (1967), denominado Método da Caminhada sobre o Contorno (Divider Method).

Essa técnica consiste na idealização de diversas caminhadas sobre o contorno primitivo da forma, $\mathrm{C}_{0}$, que encerra área $A_{0}$ e perímetro $P_{0}$, utilizando-se passos de diferentes tamanhos, $\mathrm{S}_{\mathrm{i}}$. Após a primeira caminhada, obtém-se um segundo contorno, $C_{1}$, que encerra área $A_{1}$ e perímetro $\mathrm{P}_{1}$, e assim sucessivamente.

A equação que estabelece a relação entre o perímetro $\mathrm{P}$, a extensão do passo $\mathrm{S}$ e a dimensão fractal, $\mathrm{D}_{\mathrm{f}}$, utilizada neste método, é a que se apresenta na Equação 14:

$$
P(S) \propto S^{1-D_{f}}
$$

equação que pode ser reescrita da maneira que segue:

$$
\ln P=\ln S^{1-D_{f}} \Rightarrow D_{f}=1-\frac{\ln P(S)}{\ln S}
$$

Alterando-se sucessivamente o tamanho do passo $\mathrm{S}$ entre as consecutivas caminhadas (ou iterações), obtém-se um conjunto de passos $\mathrm{S}_{\mathrm{i}}$ e correspondentes perímetros, $\mathrm{P}_{\mathrm{i}}$, a partir dos quais são computados $\ln \mathrm{S}_{\mathrm{i}}$ e $\ln P_{i}$.
A dimensão fractal, $\mathrm{D}_{\mathrm{f}}$, é obtida por meio da consideração da magnitude da inclinação $\beta_{1}$ da reta decorrente de uma regressão linear aplicada ao conjunto de pontos $\left(\ln \mathrm{S}_{\mathrm{i}}\right.$; $\left.\ln \mathrm{P}_{\mathrm{i}}\right)$, da forma que segue:

$$
\ln \hat{P}_{i}=\beta_{1} \ln S_{i}+\beta_{0}
$$

onde $\beta_{0}$ e $\beta_{1}$ são os coeficientes linear e angular da reta de regressão, respectivamente.

Observa-se que o coeficiente angular $\beta_{1}$ da reta de regressão é utilizado para a determinação da Dimensão Fractal, em conformidade com a Equação 16, que é idêntica à Equação 15:

$$
D_{f}=1-\beta_{1}
$$

Por sua vez, o coeficiente linear $\beta_{0}$, intercepto da reta de regressão com o eixo vertical $(\ln \mathrm{P})$, pode ser utilizado para a determinação de um perímetro "eficaz", $\mathrm{P}^{*}$, representativo do grupo de perímetros analisados na regressão linear, fazendo-se:

$$
P^{*}=e^{\ln \beta_{0}}
$$

A Figura 2, que será referida ao longo do texto, ilustra as iterações realizadas em processos de caminhadas sobre o contorno.

A ideia básica desse modelo foi implementada por diferentes autores. Os procedimentos mais populares são os algoritmos híbrido e exato, ambos propostos por Clark (1986). Porém, o modelo desenvolvido e ora apresentado adota uma série de premissas particulares que passam a ser descritas:

1. Admite-se que o contorno inicial da forma seja desenvolvido a partir da interligação de sucessivos segmentos retos, cujas definições se dão por meio da utilização de pontos coordenados (Figura $2-\mathrm{A}$ );

2. O tamanho do passo inicial da caminhada, $\mathrm{S}_{0}$, tem por base uma fração da extensão da menor aresta ou segmento (distância entre dois pontos consecutivos) verificado no contorno da forma. No presente modelo, a fração adotada é igual a $1 / 3$. Isso garante que todos os segmentos que compõe o contorno sejam preliminarmente considerados; 


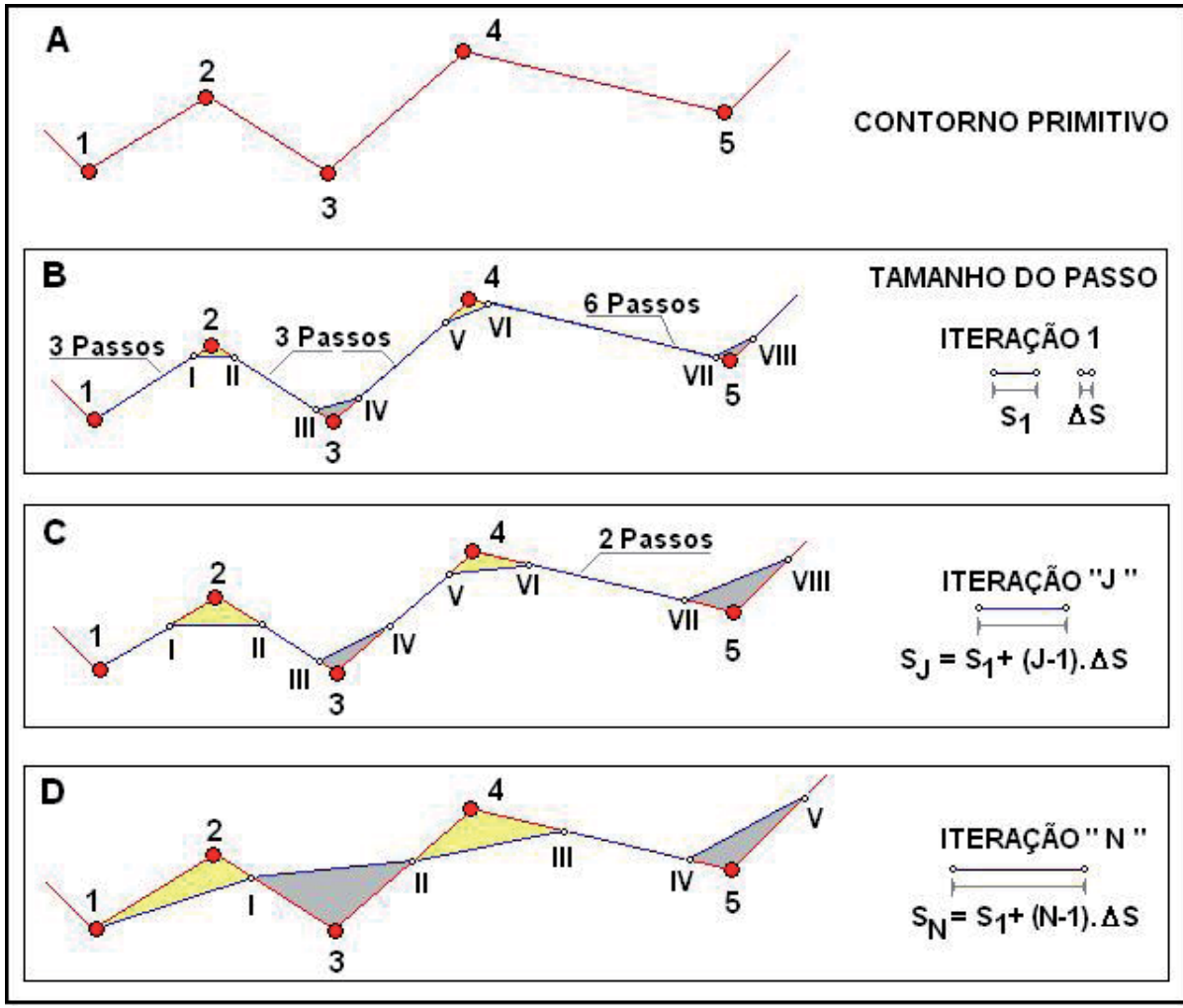

Figura 2 - Metodologia esquemática de caminhamentos sobre o contorno.

3. O comprimento do passo $\mathrm{S}_{\mathrm{i}}$ a ser utilizado na iésima iteração, é determinado em conformidade com a Equação 19:

$$
S_{i}=S_{0}+(i-1) \Delta S
$$

onde $\Delta \mathrm{S}$ é uma taxa de incremento que tem por base a extensão do passo inicial, $\mathrm{S}_{0}$. Após diversos experimentos numéricos e de maneira a evitarem-se coincidências dos pontos da caminhada com os pontos do contorno primitivo, especialmente nos casos de formas regulares, essa taxa foi arbitrada em $e / 50$ (onde $e$ é a base dos logaritmos Neperianos), o que resulta em aproximadamente $5,437 \%$ do valor do passo inicial, $\mathrm{S}_{0}$;

4. A escala $r_{i}$ é definida, em cada uma das iterações, dividindo-se o tamanho do passo $\mathrm{S}_{\mathrm{i}}$ utilizado na iteração, pelo valor do perímetro do contorno primitivo. $\mathrm{O}$ caminhamento sobre o contorno em cada uma das suas fases incrementais, ou seja, utilizando-se escalas sucessivamente maiores, é procedido por meio da utilização de passos de tamanhos constantes (Figura 2-B, C e D);

5. Mesmo que a variação da escala entre as fases incrementais ocorra de maneira uniforme, ou seja, dentro de uma progressão aritmética no que concerne ao espectro de escalas utilizado, os limites das análises procuram atender, ainda que com certa flexibilidade, aos critérios de similaridade bidimensional (BAZĂNT, 1984; BAZǍNT e PFEIFFER, 1987), por analogia aplicáveis às análises em pauta ${ }^{2}$;

6. Os diversos caminhamentos sobre o contorno não admitem a utilização de passos fracionários. Portanto, o último passo, referente ao fechamento do contorno, é simplesmente ignorado, uma vez que, inevitavelmente, não coincidirá com o ponto inicial;

7. Para uma determinada iteração (ou caminhada), o primeiro contorno a ser considerado como efetivamente

\footnotetext{
${ }^{2}$ Segundo esse critério (por analogia aplicável aos estudos aqui desenvolvidos), o incremento da escala deve ser procedido de tal maneira que a escala adotada na iteração anterior (i-1) seja a metade daquela adotada na iteração atual (i). Consequentemente, a magnitude da escala na iteração posterior (i+1) será o dobro da atual. Dessa maneira, a razão entre a escala final e a inicial, será igual a 4 . Teoricamente, a análise poderia ser encerrada com um número mínimo de três iterações assim procedidas. Entretanto, para fins de análise dos contornos das bacias hidrográficas, variações de escala compreendidas entre 3 e 4 serão entendidas como satisfatórias.
} 
válido será aquele que apresentar um número de pontos coordenados igual ou imediatamente inferior ao número de pontos que constituem o contorno primitivo. As justificativas (e exceções) para esse procedimento serão apresentadas na próxima sessão;

8. O crescimento da escala é restringido de tal maneira que a redução do número de pontos que definem o contorno da forma, em cada fase incremental, ocorra até níveis aceitáveis, evitando-se, com isso, a deterioração excessiva da forma primitiva. Esse limite (inferior), também arbitrado a partir de múltiplos estudos numéricos, foi estipulado em $12 \%$ do número de pontos coordenados que definem o contorno primitivo. Quando esse limite é alcançado, a análise é encerrada;

9. Ao final de cada procedimento incremental, a área da forma gerada deve resultar minimamente afetada, comparativamente à forma primitiva, de tal maneira que a modificação em sua magnitude possa ser entendida como desprezível (soma das regiões coloridas da Figura 2). Com base em diversos estudos numéricos, realizados a partir de uma forma fractal legítima, verificou-se que essa variação deve ser limitada a um valor percentual da magnitude da área primitiva. Essa limitação é procedida considerando-se a razão entre o número de pontos gerados na iésima iteração, $\mathrm{NPC}_{\mathrm{i}}$, com o perímetro da forma, $\mathrm{P}_{\mathrm{i}}$, e sua área, $\mathrm{A}_{\mathrm{i}}$, simultaneamente. Nesse caso, quanto maior for essa densidade, menor será a tolerância à variação da área, $\Delta \mathrm{A}_{\max } \%$, para que a magnitude da dimensão teórica seja convenientemente aproximada. Caso isso não se verifique, a etapa de cálculos é descartada e passa-se ao próximo incremento na escala.

\subsection{Considerações Referentes às Variações de Áreas}

Parece improdutivo citar o número de autores que se referem à metodologia de Richardson como inapropriada à aproximação de dimensões fractais de formas, cujos valores teóricos de $\mathrm{D}_{\mathrm{f}}$ são conhecidos. Entretanto, o que garante a aplicabilidade dessa metodologia às formas absolutamente aleatórias, a exemplo dos contornos das bacias hidrográficas, de ilhas, de continentes, etc.

Buscando um maior aprofundamento nessa questão, diversos experimentos numéricos foram procedidos por meio da utilização de formas geométricas euclidianas e fractais, objetivando a verificação da aplicabilidade em diferentes situações, tanto do método quanto do algoritmo, o que deu origem à premissa de número
9 descrita anteriormente.

No primeiro experimento numérico simulou-se um problema inverso de aproximação a uma circunferência, cujo perímetro tem dimensão fractal conhecida, coincidente com a dimensão topológica e de valor unitário. Para tanto, polígonos regulares com o número de lados gradativamente crescente, variando desde 60 até 22200 segmentos, foram utilizados.

Nesse caso, observou-se que os valores das dimensões fractais computadas apresentaram magnitudes superiores à unidade nos casos das figuras mais rugosas (com menor número de lados), entretanto convergindo coerentemente à unidade com o crescimento do número de lados.

Para o polígono de 60 lados (mais acidentado), por exemplo, obteve-se $\mathrm{D}_{\mathrm{f}}=1,00180329677552$. Por outro lado, para o polígono com 22200 lados, ou seja, uma forma dotada de contorno extremamente suave, o valor computado foi $\mathrm{D}_{\mathrm{f}}=1,00000009603594$.

Como esperado, observou-se também o crescimento coerentemente convergente das magnitudes dos perímetros e das áreas, em direção aos parâmetros da circunferência.

Por decorrer de uma análise inversa, esse conjunto de fatos em princípio valida a aplicabilidade da metodologia e do algoritmo para casos legitimamente euclidianos, sugerindo, assim, que os valores computados refletem mais apropriadamente excelentes indicadores da rugosidade da forma, ao menos do ponto de vista da geometria euclidiana.

De maneira cautelosa, Issa et al. (2003) e Blachowski e Ruebenbauer (2009), por outros caminhos também aludiram essa hipótese.

No segundo experimento numérico, estudou-se o Floco de von Koch (Figura 1), forma autenticamente fractal cuja dimensão é conhecida e tem valor $\mathrm{D}_{\mathrm{f}}=\ln$ $4 / \ln 3$. Nesse caso, pouca convergência foi verificada para cinco formas estudadas, com o número de lados (ou pontos no contorno, NPC), progressivamente crescente, variando desde 48 até 12288 .

Objetivando um estudo mais consistente, as cinco geometrias do Floco de von Koch foram analisadas por meio da consideração da variação percentual da área gerada em cada iteração, relativamente à área primitiva da forma. Para tanto, diversos limites admissíveis e progressivos de variação percentual da área, $\Delta \mathrm{A}_{\max } \%$, foram estipulados e cada uma das geometrias foi verificada 
com esses limites, relativamente ao valor teórico de $\mathrm{D}_{\mathrm{f}}$

Esse enfoque possibilitou a construção de uma curva de exclusão ou aceitação de uma dada iteração, a partir dos valores de $\Delta \mathrm{A}_{\max } \%$ que melhor aproximaram o valor teórico de $\mathrm{D}_{\mathrm{f}}$, para as cinco formas analisadas. Seguramente, a variável central nessa questão é o número de pontos no contorno, NPC, que varia substancialmente entre formas progressivamente mais complexas.

Objetivando tornar o problema independente do sistema de unidades adotado (inclusive dos seus múltiplos ou submúltiplos), associações isoladas desse escalar às áreas ou aos perímetros, foram evitadas. Assim, ambas as variáveis $(\mathrm{P} \mathrm{e} \mathrm{A})$ foram associadas ao número de pontos no contorno, NPC, simultaneamente, por meio da constante de proporcionalidade euclidiana $\mathrm{k}$, que é uma grandeza adimensional (Equação 3, rearranjada).

Com isso, a curva de exclusão/aceitação da iteração foi escrita sob a forma ( $\mathrm{NPC} / \mathrm{k}$ versus $\Delta \mathrm{A}_{\max } \%$ ) que aparenta ser a maneira mais razoável de considerarem-se os três parâmetros da forma, simultaneamente. A curva de exclusão de iterações, que relaciona a densidade de pontos no contorno, $\mathrm{NPC}_{\mathrm{i}}$, e a variação percentual admissível da área, $\Delta \mathrm{A}_{\max } \%$, relativamente à área primitiva, é a que se apresenta na Figura 3.

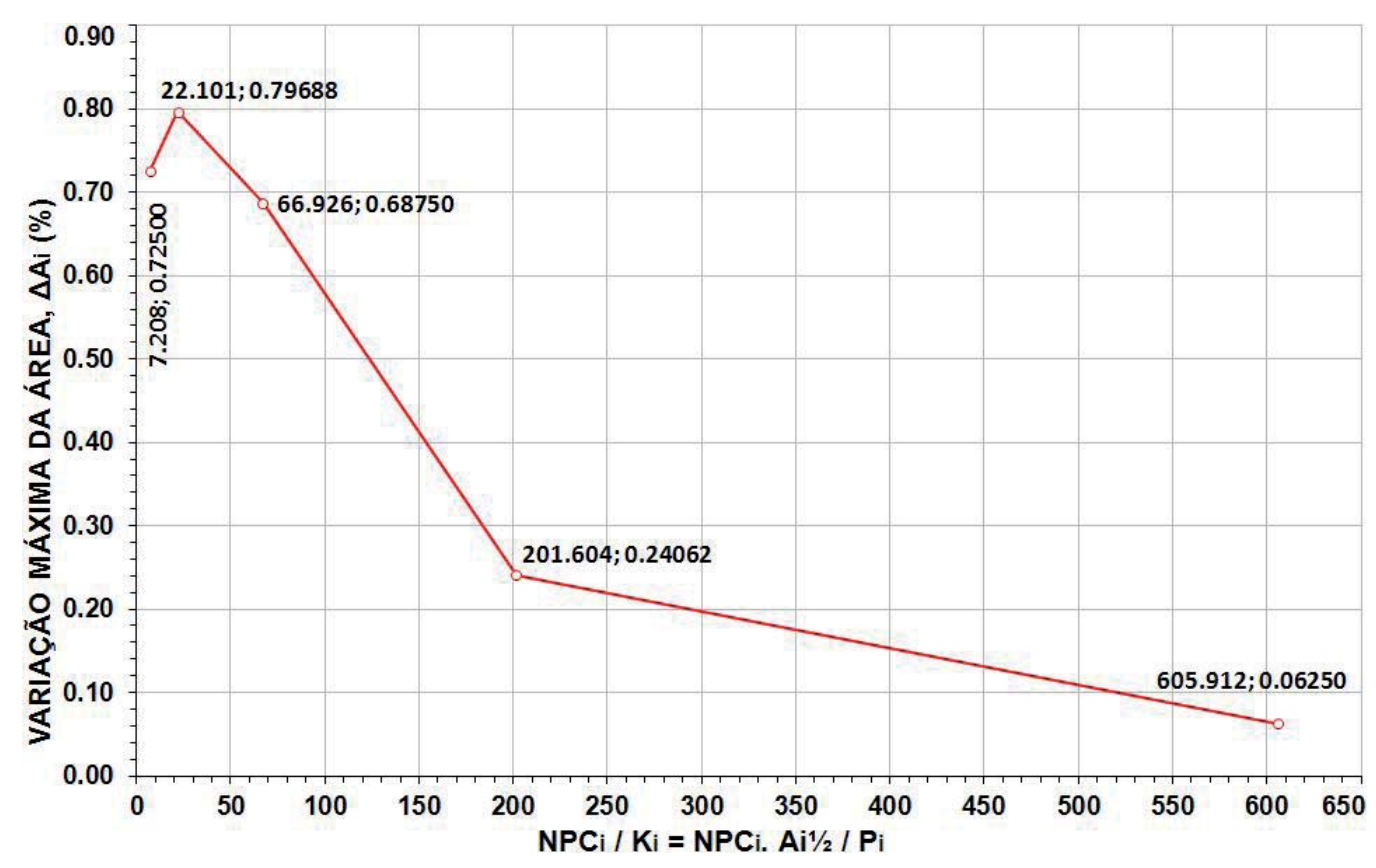

Figura 3 - Curvas de exclusão de iterações $\triangle A_{\max } \%$ versus Densidade Linear de NPC/k.

Os erros percentuais em Df decorrentes da aplicação do algoritmo, sem e com a aplicação do critério de limitação da variação da área à aproximação das dimensões fractais das cinco formas estudadas, ao valor teórico $\mathrm{D}_{\mathrm{f}}=\ln 4 / \ln 3$, são apresentados na Figura 4, em função da densidade linear de pontos no contorno, $\mathrm{NPCi} / \mathrm{k}$.

A aplicabilidade desse critério à análise de bacias hidrográficas e de outras formas correlatas encontra suporte em estudos desenvolvidos por Clarke e Schweizer (1991), apud Klinkemberg (1994).

Nesses estudos, constataram os autores que o simples escalonamento anisotrópico (realizado em uma das direções) de uma forma primitivamente autossimilar e que a transforma em outra, autoafim (a exemplo do escalonamento unidirecional que transforma uma forma circunferencial em uma forma elíptica), afeta muito pouco ou em quase nada a dimensão fractal, $D_{f}$, mesmo que o escalonamento aplicado seja da ordem de $10^{2}$.

Ora, a forma do floco de von Koch, quando altamente densa, "aproxima-se" de uma circunferência. Se escalonada, nas direções horizontal ou vertical, essa forma circunferencial tenderá à forma elíptica, com a sua dimensão fractal praticamente inalterada. Observa-se que ambas as formas citadas são comuns e geometricamente aproximáveis em estudos de bacias hidrográficas. 


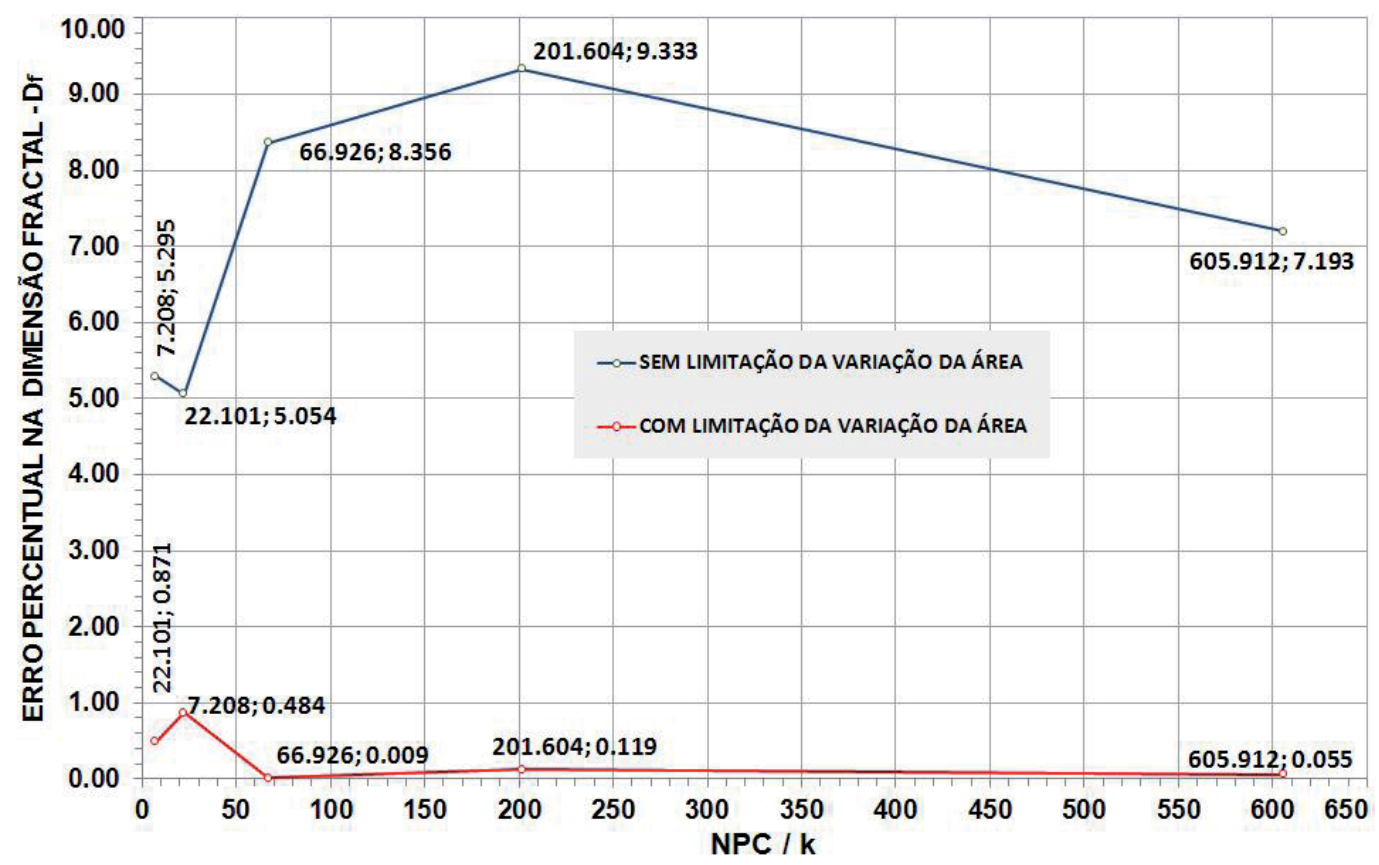

Figura 4 - Gráfico Erros \%em Df versus NPC/k, com e sem limitações da variação da área.

\section{Estudo Computacional Preliminar}

Para o desenvolvimento das análises que seguem utilizou-se o software FloRiSys (FERREIRA, 2014) que é um conjunto de programas desenvolvido para a análise de riscos de natureza hidrológica e que inclui módulos destinados à determinação e ao tratamento de informações morfométricas associadas às bacias hidrográficas.

Com o objetivo preliminar de aplicar-se a metodologia em discussão, analisou-se a bacia hidrográfica do Rio Ipojuca localizada no Estado de Pernambuco, região nordeste do Brasil.

Trata-se de uma bacia de porte intermediário com forma alongada na direção do seu eixo principal e contorno significativamente irregular, situada em relevo relativamente dissecado. $O$ contorno dessa bacia, juntamente com a rede de drenagem natural, são esquematicamente ilustrados na Figura 5 (Fonte: SUDENE-ITEP; Governo do Estado do Pernambuco).

De maneira a obter-se um panorama geral da curva $\ln \mathrm{P}$ versus $\ln \mathrm{S}$ (Equação 14), e a título de ilustraremse os critérios referidos, as premissas anteriormente

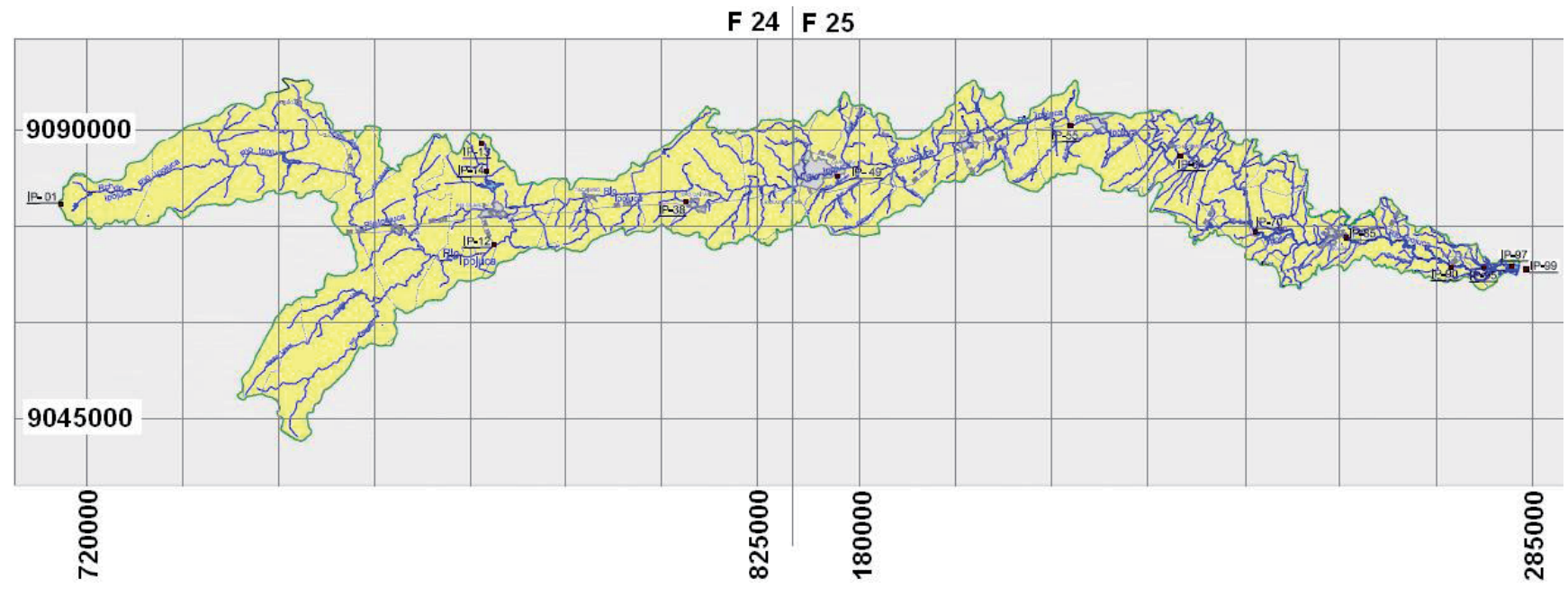

Figura 5 - Delimitação da Bacia Hidrográfica do Rio Ipojuca e rede de drenagem. 
apresentadas são preliminarmente relaxadas. Dessa maneira, não se impuseram quaisquer limitações relativamente aos números máximos e mínimos de pontos gerados em cada iteração do algoritmo, relativamente ao número primitivo de pontos coordenados, bem como não se invalidaram iterações em virtude de variações das áreas das formas geradas nas diversas iterações, relativamente à área primitiva.
Para tanto, essa primeira análise foi levada a efeito utilizando-se o passo inicial previsto na premissa de número 3 da sessão 4 e um número arbitrário de 3000 iterações do algoritmo. A Figura 6 mostra a reta decorrente da regressão linear (Equação 14), os parâmetros resultantes da regressão e a dimensão fractal, $\mathrm{D}_{\mathrm{f}}$, calculada em conformidade com a Equação 15.

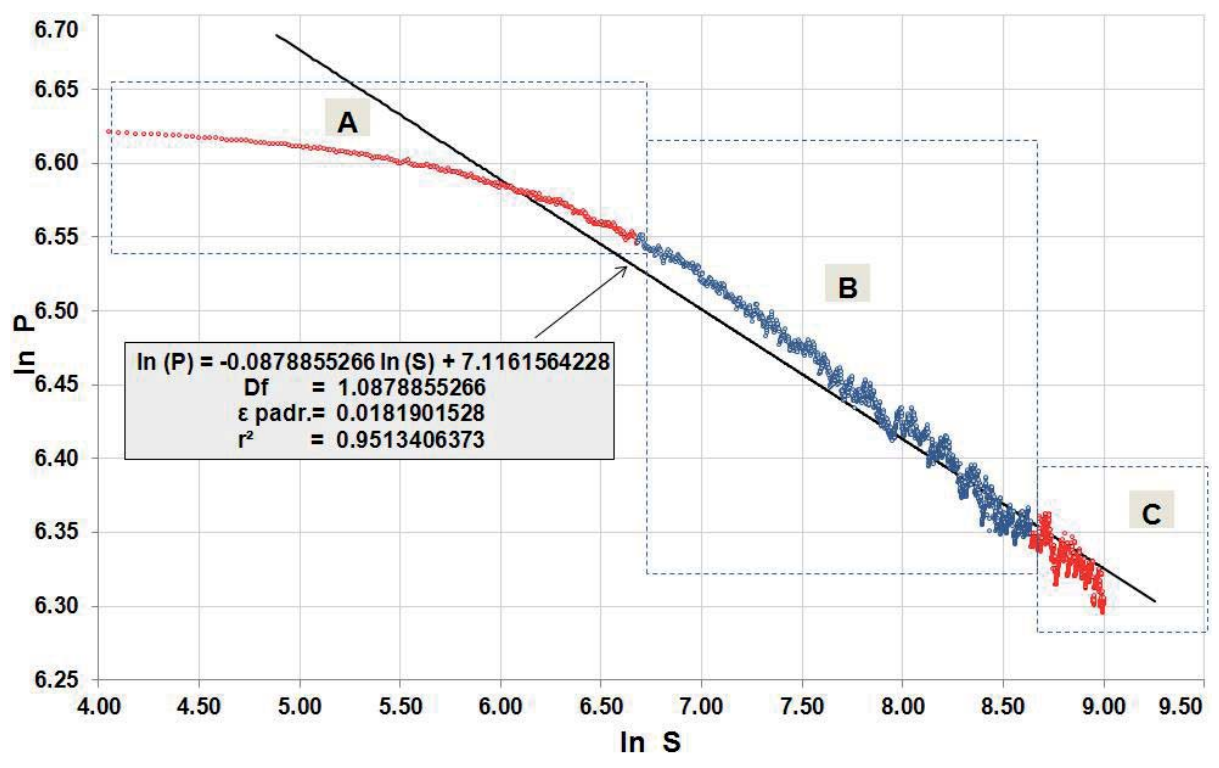

Figura 6 - Curva ln P versus ln S e regressão linear para o conjunto de 3000 iterações do algoritmo.

Como se observa na Figura 6, o gráfico resultante foi subdividido em três regiões identificadas pelas letras A, B e C. Da análise desse gráfico depreende-se, inicialmente, o comportamento altamente não linear do conjunto de pontos situados na região A. Nessa região, os tamanhos dos passos utilizados são ainda bastante pequenos, fazendo com que o caminhamento sobre um mesmo seguimento do contorno ocorra por meio da utilização de diversos passos (Figura 2, b e c).

Consequentemente, a variação do perímetro entre sucessivas iterações será muito pequena. Por outro lado, as escalas, decorrentes dos tamanhos dos passos utilizados, crescem linearmente dentro de uma progressão aritmética, portanto mais rapidamente que as variações dos perímetros, entre as iterações.

Como as iterações seguintes ainda se dão com passos pequenos dentro dessa região, a não linearidade fica claramente evidenciada (entretanto progressivamente menor) até o limite entre as regiões $\mathrm{A}$ e $\mathrm{B}$. Como consequência, a inclinação da reta de regressão apresentará um coeficiente angular com menor inclinação, diminuindo, artificialmente, o valor numérico da Dimensão Fractal, $\mathrm{D}_{\mathrm{f}^{\prime}}$ Esse fato foi igualmente discutido por Klinkemberg (1994).

Observou-se também, que o limite entre as regiões A e B se verifica quando o número de pontos gerados para a constituição do contorno da iésima iteração aproxima-se do número de pontos que compõe o contorno primitivo da forma.

A região $\mathrm{C}$, por outro lado, evidencia certo distúrbio de regularidade nas respostas, no que diz respeito aos contornos originados, fato atribuído ao número reduzido de pontos coordenados produzidos para a formação dos contornos nas iterações finais do algoritmo, com consequente descaracterização (progressiva) da forma primitiva. Conforme referido, diversos ensaios numéricos demonstraram que esse fato passa a ocorrer de maneira mais pronunciada, em contornos gerados com um número de pontos inferior a $12 \%$, aproximadamente, do número de pontos que constituem o contorno primitivo. 


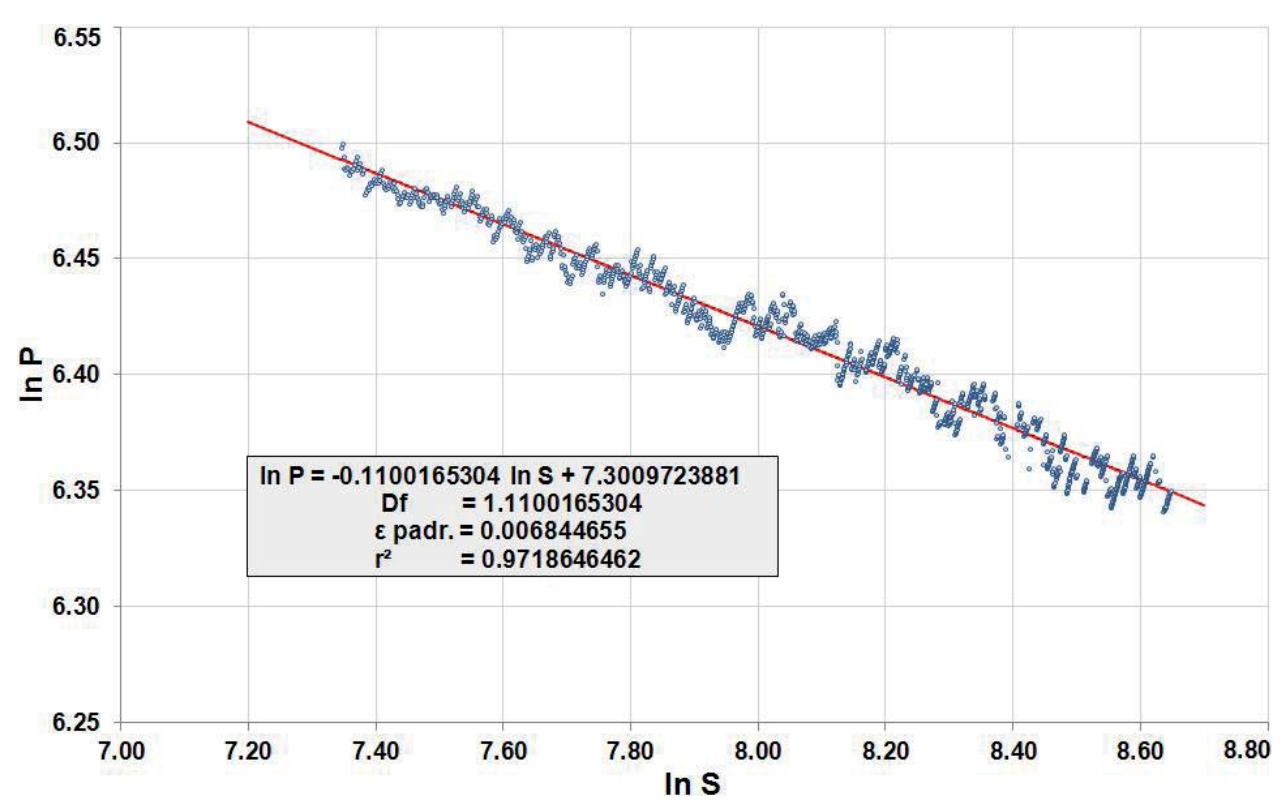

Figura 7 - Regressão linear para o conjunto de pontos situados na região B da Figura 6.

As breves considerações expostas justificam, portanto, a exclusão dos pontos situados nas regiões A e C, conforme premissas de números 7 e 8 , anteriormente apresentadas.

Adotando-se as premissas enunciadas, uma nova regressão correspondente à região central $\mathrm{B}$, foi realizada. A Figura 7 ilustra a reta decorrente da regressão linear (Equação 16), e seus parâmetros, assim como a dimensão fractal, $\mathrm{D}_{\mathrm{f}}$, calculada por meio da Equação 17.

\subsection{Análise Inversa para a Determinação do Contorno Regularizado e do Perímetro a ele Associado.}

Da observação da reta de regressão apresentada na Figura 7 e do conjunto de pontos $\ln \mathrm{P}_{\mathrm{i}}$ versus $\ln \mathrm{S}_{\mathrm{i}}$ que a originam, depreende-se a possibilidade de existência de um certo contorno $\mathrm{C}_{\mathrm{f}}$, aqui denominado "contorno regularizado", gerado com um passo de tamanho $\mathrm{S}_{\mathrm{f}}$, na escala $E_{f}$ e com perímetro $P_{f}$, que represente de maneira satisfatória e em termos médios, o conjunto de perímetros determinados nas análises.

Esse contorno deve decorrer do equilíbrio entre as variáveis abordadas, que afetam a dimensão fractal da forma.

Naturalmente, além da área $\mathrm{A}_{\mathrm{i}}$, também o perímetro $\mathrm{P}_{\mathrm{i}}$ associado ao contorno $\mathrm{C}_{\mathrm{i}}$ é uma função do número de pontos coordenados que o constitui, $\mathrm{NPC}_{i}$. Por sua vez, esse escalar é uma função do tamanho do passo
$\mathrm{S}_{\mathrm{i}}$ empregado na iteração. Portanto, o equacionamento do problema poderia ser restringido às análises com ferramentas do cálculo funcional. Entretanto, esse tipo de equacionamento da solução mostrar-se-ia pouco computacional.

Uma maneira de contornar-se a questão parece ser a associação dessas variáveis em espaços normalizados.

Isso pode ser feito de maneira relativamente simples, aproveitando-se os pares $\ln \mathrm{P}_{\mathrm{i}}$ e $\ln \mathrm{S}_{\mathrm{i}}$ produzidos ao longo do processo iterativo (além de $\ln \mathrm{NP}_{\mathrm{i}}$ ), para o cômputo de pares normalizados $\left(\ln \mathrm{P}_{\mathrm{i}} / \ln \mathrm{P}_{\max } ; \ln \mathrm{S}_{\mathrm{i}} / \ln \right.$ $\left.\mathrm{S}_{\text {max }}\right)$ e $\left(\ln \mathrm{NP}_{\mathrm{i}} / \ln \mathrm{NP}_{\max } ; \ln \mathrm{S}_{\mathrm{i}} / \ln \mathrm{S}_{\max }\right)$, onde $\mathrm{P}_{\max }, \mathrm{S}_{\max } \mathrm{e}$ $\mathrm{NP}_{\max }$ são os valores máximos referentes ao perímetro, ao tamanho do passo e ao número de pontos alcançados no conjunto de iterações, respectivamente.

A partir do conjunto de informações normalizadas, duas novas regressões relacionando o número de pontos no contorno, NPC, e o passo $\mathrm{S}$, ao perímetro $\mathrm{P}$, são levadas a efeito de maneira a gerar novas equações, conforme segue:

$$
\frac{\ln P_{i}}{\ln P_{\max }}=\beta_{1 \_} s \frac{\ln S_{i}}{\ln S_{\max }}+\beta_{0 \_s} \quad \rightarrow \frac{\ln S_{i}}{\ln S_{\max }}=\left(\frac{\ln P_{i}}{\ln P_{\max }}-\beta_{0 \_s}\right) \frac{1}{\beta_{1 \_} s}
$$

$$
\frac{\ln P_{i}}{\ln P_{\max }}=\beta_{1 \_N P} \frac{\ln N P_{i}}{\ln N P_{\max }}+\beta_{0_{-} N P} \rightarrow \frac{\ln N P_{i}}{\ln N P_{\max }}=\left(\frac{\ln P_{i}}{\ln P_{\max }}-\beta_{0_{-} N P}\right) \frac{1}{\beta_{1 \_N P}}
$$


onde os subscritos $\mathrm{S}$ e NP, referem-se ao passo $\mathrm{S}$ e ao número de pontos no contorno, NPC.

Igualando-se os segundos membros das Equações 20 e 21 , nas suas segundas formas, obtêm-se o ponto de intersecção das retas, o que torna possível determinar o valor teórico do perímetro regularizado, $\mathrm{P}_{\mathrm{t}}$ :

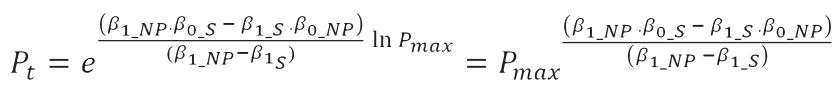

Substituindo-se na Equação 16 o valor de $\mathrm{P}_{\mathrm{t}}$ assim verificado, computa-se a magnitude teórica do passo $\mathrm{S}_{\mathrm{t}}$ que conduzirá ao valor desse perímetro, em conformidade com a Equação 23:

$$
S_{t}=e^{\frac{\ln P_{f}-\beta_{0}}{\beta_{1}}}
$$

Para o problema em análise, as retas decorrentes das regressões lineares realizadas no espaço normal, assim como suas equações, são apresentadas na Figura 8.

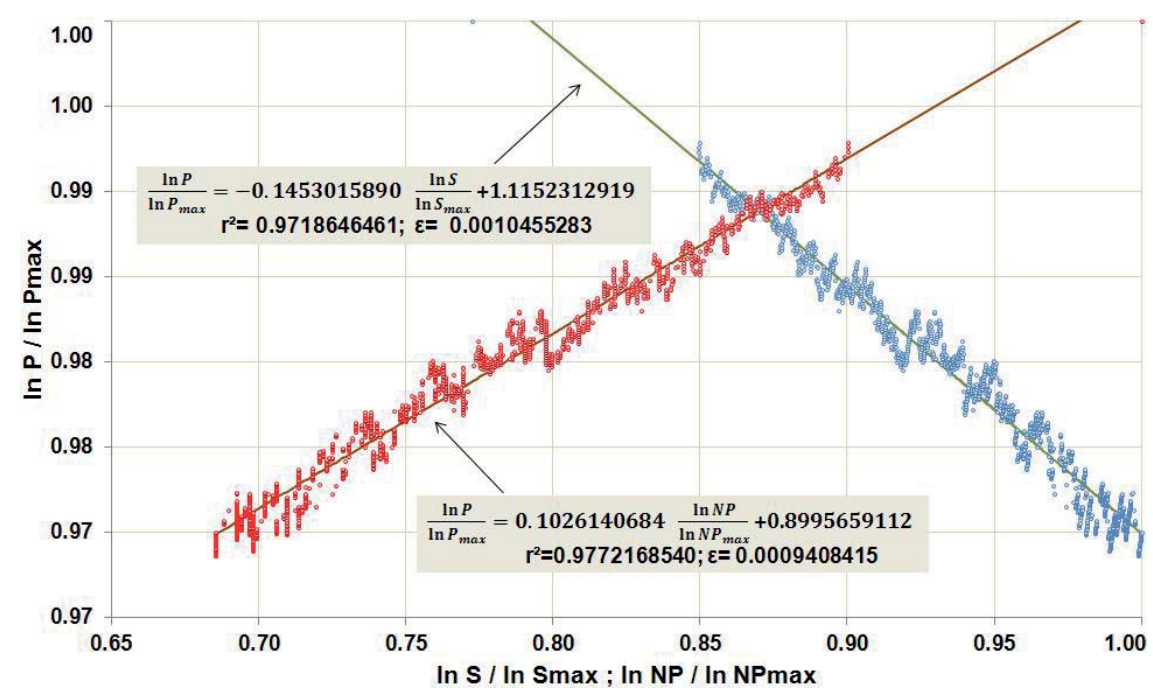

Figura 8 - Regressões lineares no espaço normalizado.

Para o problema em análise foram determinados os valores teóricos que seguem:

- Perímetro Teórico, $\mathrm{P}_{\mathrm{t}}$ : 647,735656km (Equação 20);

- Tamanho Teórico do Passo, $\mathrm{S}_{\mathrm{t}}: 1,847343$ km (Equação 21).

De posse dessas informações, uma última aplicação do algoritmo é procedida, obtendo-se os valores finais que seguem:

- Escala, $\mathrm{E}_{\mathrm{f}}$ :

- Perímetro, $\mathrm{P}_{\mathrm{f}}$ : $650,583836 \mathrm{~km}$;

- Área, $\mathrm{A}_{\mathrm{f}}$ : $3455,051488 \mathrm{~km}^{2}$.

Observa-se que, para a determinação da dimensão fractal para o contorno regularizado assim computado, basta reprocessar o algoritmo para os pontos coordenados referentes a esse contorno. A Figura 9 ilustra o contorno primitivo da bacia, grafado juntamente com o contorno regularizado, ambos girados e plotados em um sistema coordenado arbitrário.

Como se observa, os valores teóricos de $\mathrm{P}_{t}$ e $\mathrm{S}_{\mathrm{t}}$ determinados em conformidade com as Equações 22 e 23, conduzirão à perímetros finais de magnitudes aproximadas, quando da última execução do algoritmo. Duas são as explicações para esse fato:

- As equações referidas têm origens em regressões lineares, portanto são aproximações teóricas de natureza estatística;

- Os números de pontos nos contornos, obtidos em iterações sucessivas do algoritmo, não variam linearmente com os tamanhos dos passos ou escalas adotadas.

Esses fatos desobrigam, portanto, a coincidência absoluta entre os valores dos perímetros teóricos e daquele obtido no processamento final do algoritmo, raciocínio que se aplica, igualmente, ao número de pontos no contorno, NPC.

A Tabela 1 apresenta uma comparação dos principais indicadores morfométricos de compacidade, computados com os perímetros primitivos e regularizado. 


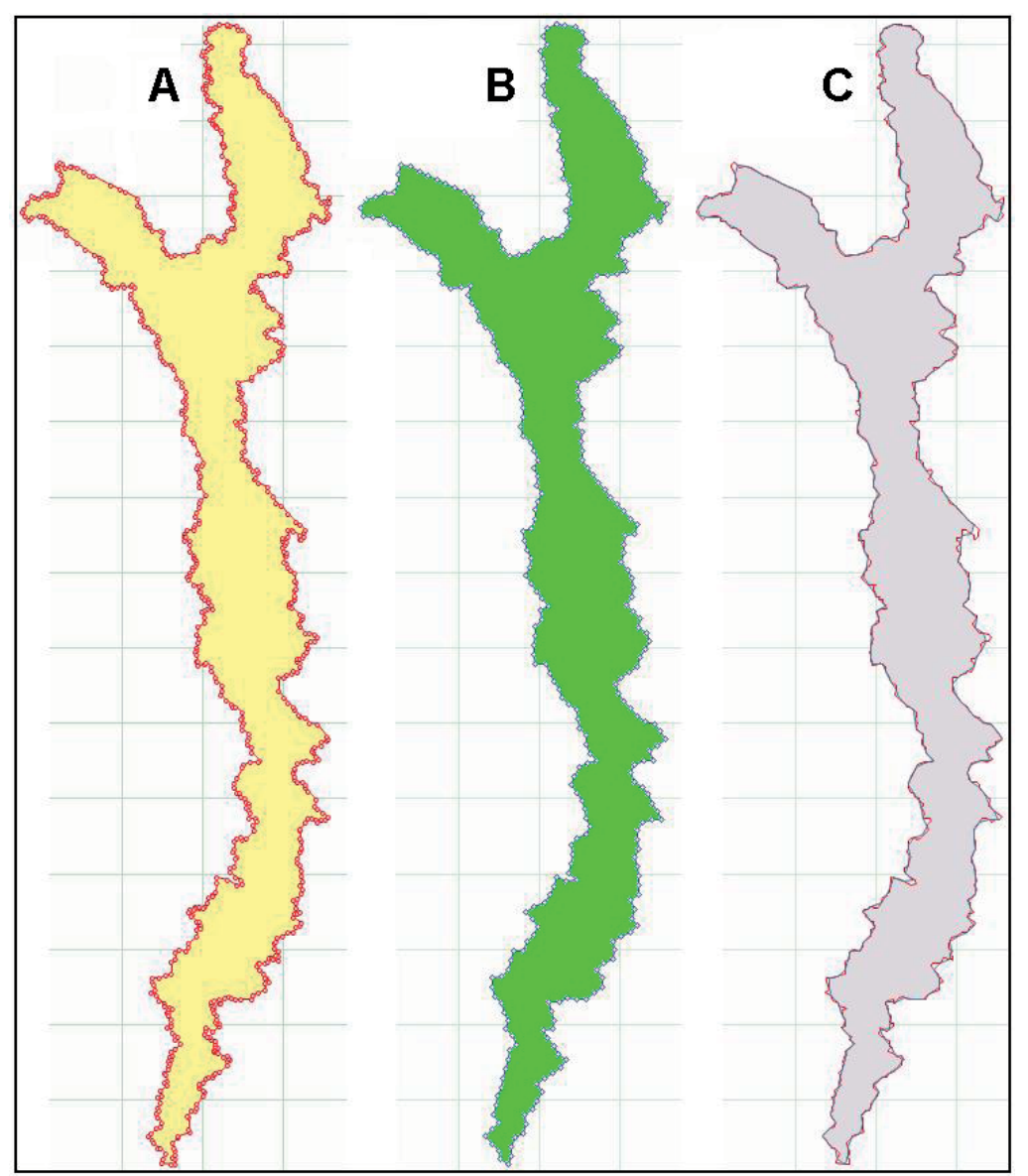

Figura 9 - Contornos: Primitivo (A), Regularizado (B) e Sobrepostos (C); Sistema arbitrário - $m$.

Tabela 1: Indicadores morfométricos para a bacia hidrográfica do Rio Ipojuca.

\begin{tabular}{|c|c|c|c|c|c|c|c|c|}
\hline CONTORNO & PONTOS & $\begin{array}{l}\text { PERÍMETRO-P } \\
(\mathrm{km})\end{array}$ & $\begin{array}{l}\text { ÁREA-A } \\
\left(\mathrm{km}^{2}\right)\end{array}$ & $\triangle A(\%)$ & $\Delta \mathrm{P}(\%)$ & $\begin{array}{c}\text { kc - íND. DE } \\
\text { COMPACIDADE }\end{array}$ & $\begin{array}{l}\text { RC- RAZÃ̃O DE } \\
\text { CIRCULARIDADE }\end{array}$ & $\begin{array}{c}\text { CONSTANTE } \\
\text { EUCUIDIANA-K }\end{array}$ \\
\hline PRIMITIVO & 842 & 755.7277 & 3453.3714 & \multirow{2}{*}{0.0486} & \multirow{2}{*}{-16.1615} & 3.6278 & 0.0760 & 12.8601 \\
\hline REGULARIZADO & 352 & 650.5838 & 3455.0515 & & & 3.1223 & 0.1026 & 11.0682 \\
\hline
\end{tabular}

\subsection{Breves Comentários aos Resultados Preliminares}

De acordo com o que se depreende das imagens apresentadas na Figura 9, o contorno primitivo da forma (9A) fica satisfatoriamente resguardado (9B). Da mesma maneira e em conformidade com as informações apresentadas na Tabela 1 , a modificação verificada na magnitude da área é desprezível.

Entretanto, a redução introduzida tanto do número de pontos que formam o novo contorno, como na magnitude do perímetro a ele associado, é drástica, com evidente reflexo sobre os valores dos descritores morfométricos de compacidade.

\section{Estudo Computacional de Bacias Hidrográficas Brasileiras}

Com o objetivo de avaliar os parâmetros morfométricos de bacias hidrográficas, treze bacias de diferentes portes e características, foram selecionadas. As bacias hidrográficas analisadas, suas características geométricas e informações referentes às suas localizações, são apresentadas na Tabela 2.

As bacias dos Rios Capibaribe, Una, Ipojuca, Goiana, Sinhaém, Jaboatão-Pirapama, Botafogo-Igarassú e Arimbí-Tapera-Merepê, estão localizadas no estado do Pernambuco (PE), região nordeste do 
Brasil. No mesmo Estado e região, encontra-se a bacia do Açude da Cachoeira II, já examinada por Ferreira et al. (2010) sob o enfoque convencional.

A bacia do Rio São João, estudada por Salles (2010) e situada no Estado de Minas Gerais, MG, além de outra com o mesmo nome, entretanto localizada em Carambeí, Estado do Paraná (PR), discutida por Santos et al. (2012) e aqui diferenciada pela letra A, são analisadas. Estuda-se também, a bacia do Rio São Bartolomeu (SENA-SOUZA et al., 2013), que compreende regiões do Estado de Goiás e do Distrito Federal.

Com o fito de mais bem avaliar-se a aplicabilidade das rotinas computacionais desenvolvidas, assim como o espectro de aplicabilidade do modelo de análise fractal, a menor delas foi estudada também relativamente às suas sub-bacias.
Trata-se da bacia hidrográfica do Riacho da Cachoeira das Pombas, localizada no Município de Guanhães, MG, examinada por Tonello (2005).

Para efeitos de maior rigor de análise, essa bacia foi discretizada pela autora em cinco sub-bacias e uma área comum a elas, designada interbacia, em conformidade com a geomorfologia associada aos canais naturais de drenagem. A microbacia, assim como as suas sub -bacias são esquematicamente mostradas na Figura 10. As características geométricas básicas desse conjunto são apresentadas na Tabela 3. Em todos os casos, as áreas e perímetros iniciais foram recalculados com a adoção de critério único. Portanto, pequenas diferenças relativamente aos valores determinados pelos autores citados, foram inevitáveis.

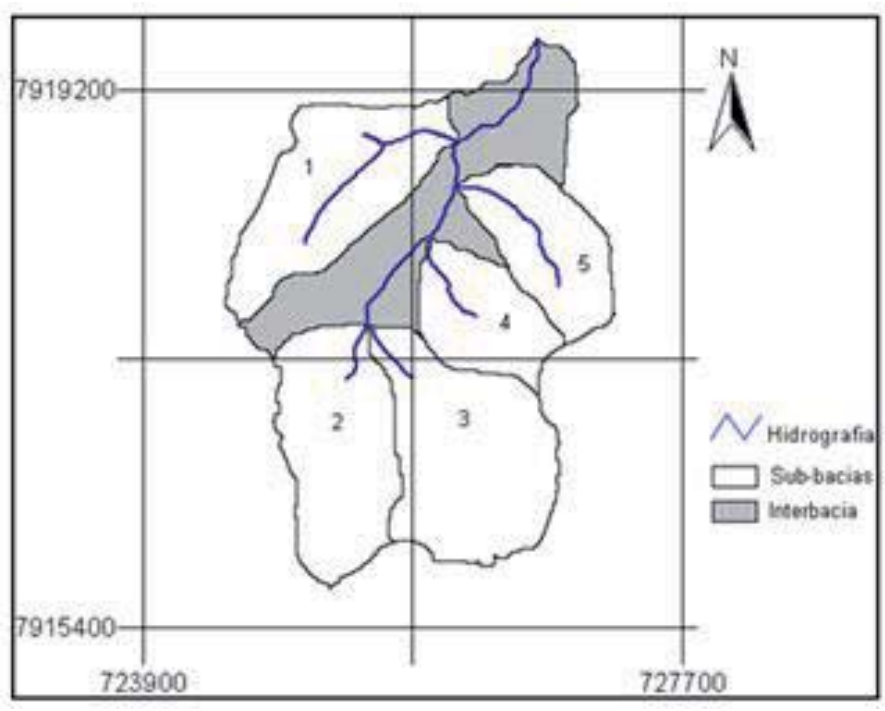

Fonte: TONELLO (2005)

Figura 10 - Bacia hidrográfica do Riacho da Cachoeira das Pombas e suas sub-bacias.

Tabela 2: Relação de bacias hidrográficas analisadas, ordenada pela magnitude da área de drenagem.

\begin{tabular}{|c|c|c|c|c|c|c|c|}
\hline \multirow{3}{*}{ BACIA } & \multirow{3}{*}{ ESTADO } & \multicolumn{4}{|c|}{ REGIÃO DE LOCALIZAÇÃO } & \multirow{2}{*}{\multicolumn{2}{|c|}{$\begin{array}{l}\text { ÁREA DE } \\
\text { DRENAGEM PERÍMETRC }\end{array}$}} \\
\hline & & \multicolumn{2}{|c|}{ INICIAL } & \multicolumn{2}{|c|}{ FINAL } & & \\
\hline & & LAT. (S) & LONG. (W) & LAT. (\$) & LONG. (W) & $\left(\mathrm{km}^{2}\right)$ & $(\mathrm{km})$ \\
\hline RIO CAPIBARIBE & PE & $07^{\circ} 40^{\prime} 13^{\prime \prime}$ & $34^{\circ} 47^{\prime} 30^{\prime \prime}$ & $08^{\circ} 22^{\prime} 38^{\prime \prime}$ & $36^{\circ} 42^{\prime} 32^{\prime \prime}$ & 7377.689 & 614.349 \\
\hline RIO UNA & PE & $08^{\circ} 15^{\prime} 59^{\prime \prime}$ & ' $35^{\circ} 05^{\prime} 17^{\prime \prime}$ & $09^{\circ} 00^{\prime} 00^{\prime \prime}$ & $36^{\circ} 43^{\prime} 39^{\prime \prime}$ & 6740.166 & 579.272 \\
\hline RIO SÃO BARTOLOMEU & GO-DF & $15^{\circ} 36^{\prime} 05^{\prime \prime}$ & $48^{\circ} 06^{\prime} 36^{\prime \prime}$ & $16^{\circ} 52^{\prime} 01^{\prime \prime}$ & $47^{\circ} 30^{\prime} 17^{\prime \prime}$ & 5537.125 & 493.759 \\
\hline RIOIPOJUCA & PE & $07^{\circ} 22^{\prime} 05^{\prime \prime}$ & $34^{\circ} 48^{\prime} 09^{\prime \prime}$ & $07^{\circ} 55^{\prime} 05^{\prime \prime}$ & $35^{\circ} 41^{\prime} 31^{\prime \prime}$ & 3453.371 & 755.728 \\
\hline RIO GOIANA & PE & $07^{\circ} 22^{\prime} 05^{\prime \prime}$ & $35^{\circ} 41^{\prime} 31^{\prime \prime}$ & $07^{\circ} 55^{\prime} 05^{\prime \prime}$ & $34^{\circ} 48^{\prime} 09^{\prime \prime}$ & 2879.616 & 289.759 \\
\hline RIO SINHAEEM & PE & $08^{\circ} 13^{\prime} 07^{\prime \prime}$ & $35^{\circ} 00^{\prime} 00^{\prime \prime}$ & $08^{\circ} 44^{\prime} 20^{\prime \prime}$ & $35^{\circ} 51^{\prime} 01^{\prime \prime}$ & 2088.742 & 327.858 \\
\hline RIOS JABOATÃO-PIRAPAMA & PE & $08^{\circ} 00^{\prime} 30^{\prime \prime}$ & $35^{\circ} 23^{\prime} 36^{\prime \prime}$ & $08^{\circ} 24^{\prime} 54^{\prime \prime}$ & $34^{\circ} 51^{\prime} 22^{\prime \prime}$ & 1255.372 & 202.538 \\
\hline RIOS BOTAFOGO-IGARASSÚ & PE & $07^{\circ} 40^{\prime} 34^{\prime \prime}$ & $38^{\circ} 29^{\prime} 59^{\prime \prime}$ & $07^{\circ} 58^{\prime} 50^{\prime \prime}$ & $38^{\circ} 14^{\prime} 47^{\prime \prime}$ & 1063.498 & 214.500 \\
\hline RIO DO AÇUDE DA CACHOEIRA ॥ & PE & $07^{\circ} 40^{\prime} 32^{\prime \prime}$ & $38^{\circ} 29^{\prime} 58^{\prime \prime}$ & $07^{\circ} 40^{\prime} 33^{\prime \prime}$ & $38^{\circ} 29^{\prime} 59^{\prime \prime}$ & 394.558 & 113.980 \\
\hline RIO SÃO JOÃO & MG & $20^{\circ} 04^{\prime} 03^{\prime \prime}$ & $43^{\circ} 43^{\prime} 41^{\prime \prime}$ & $19^{\circ} 53^{\prime} 10^{\prime \prime}$ & $43^{\circ} 27^{\prime} 43^{\prime \prime}$ & 182.183 & 83.499 \\
\hline RIO SÃO JOÃO - A & PR & $24^{\circ} 46^{\prime} 53^{\prime \prime}$ & $50^{\circ} 14^{\prime} 18^{\prime \prime}$ & $24^{\circ} 58^{\prime} 11^{\prime \prime}$ & $50^{\circ} 01^{\prime} 55^{\prime \prime}$ & 145.956 & 69.437 \\
\hline RIOS ARIMBÍ-TAPERA-MEREPÊ & PE & $08^{\circ} 23^{\prime} 17^{\prime \prime}$ & " $35^{\circ} 09^{\prime} 11^{\prime \prime}$ & $08^{\circ} 34^{\prime} 15^{\prime \prime}$ & $34^{\circ} 56^{\prime} 51^{\prime \prime}$ & 128.888 & 65.465 \\
\hline R. DA CACHOEIRA DAS POMBAS & MG & $18^{\circ} 48^{\prime} 23^{\prime \prime}$ & $42^{\circ} 52^{\prime} 31^{\prime \prime}$ & $18^{\circ} 50^{\prime} 25^{\prime \prime}$ & $42^{\circ} 50^{\prime} 20^{\prime \prime}$ & 7.009 & 12.666 \\
\hline
\end{tabular}


Tabela 3: Sub-bacias da bacia hidrográfica do Riacho da Cachoeira das Pombas.

\begin{tabular}{|c|c|c|c|c|c|c|}
\hline \multirow{3}{*}{$\begin{array}{l}\text { SUB-BACIAS DA BACIA DO RIACHO } \\
\text { DA CACHOEIRA DAS POMBAS }\end{array}$} & \multicolumn{4}{|c|}{ REGIÃO DE LOCALIZAÇÃ̃O } & \multirow{2}{*}{\multicolumn{2}{|c|}{$\begin{array}{c}\text { ÁREA DE } \\
\text { DRENAGEM PERÍMETRO }\end{array}$}} \\
\hline & \multicolumn{2}{|c|}{ INICIAL } & \multicolumn{2}{|c|}{ FINAL } & & \\
\hline & LAT. (S) & LONG. (W) & LAT. (S) & LONG. (W) & $\left(\mathrm{km}^{2}\right)$ & $(\mathrm{km})$ \\
\hline SUB-BACIA 01 & \multirow{6}{*}{$18^{\circ} 48^{\prime} 23^{\prime \prime}$} & \multirow{6}{*}{$42^{\circ} 52^{\prime} 31^{\prime \prime}$} & \multirow{6}{*}{$18^{\circ} 50^{\prime} 25^{\prime \prime}$} & \multirow{6}{*}{$42^{\circ} 50^{\prime} 20^{\prime \prime}$} & 1.269 & 5.642 \\
\hline SUB-BACIA 02 & & & & & 1.248 & 5.163 \\
\hline SUB-BACIA 03 & & & & & 1.511 & 5.655 \\
\hline SUB-BACIA 04 & & & & & 0.684 & 3.661 \\
\hline SUB-BACIA OS & & & & & 0.786 & 3.916 \\
\hline INTERBACIA & & & & & 1.519 & 9.352 \\
\hline
\end{tabular}

\section{Resultados Obtidos}

No que segue, os principais resultados obtidos passam a ser apresentados. Esse conjunto de resultados reúne informações relativas à metodologia aplicada, assim como referentes aos principais descritores de compacidade relativos às bacias hidrográficas estudadas.

\subsection{Análises de Regressão}

As informações referentes às regressões lineares levadas a efeito são mostradas nas Tabelas 4 e 5, juntamente com as dimensões fractais computadas. Essas tabelas trazem, complementarmente, as razões $\mathrm{r}_{\mathrm{f}} / \mathrm{r}_{\mathrm{i}}$ entre as escalas finais e iniciais atingidas nas análises, considerando-se as restrições superiores e inferiores relativas aos números de pontos no contorno, $\mathrm{NPC}_{\mathrm{i}}$, assim como aquela referente à variação da área, $\Delta \mathrm{A}_{\max } \%$. Por questões de mera conveniência, as vírgulas decimais foram substituídas por pontos decimais.

\subsection{Descritores Morfométricos de Compacidade}

As variações dos principais descritores de compacidade aqui analisados, quais sejam, o Índice de Compacidade de Gravelius, $\mathrm{k}_{\mathrm{C}}$, e a Razão de Circularidade de Miller, $\mathrm{R}_{\mathrm{C}}$, decorrentes das modificações introduzidas nas magnitudes dos perímetro associados aos contornos da bacias, passam a ser apresentados nas Tabelas 6 e 7 . Observe-se que, também nesse caso, as vírgulas decimais foram substituídas por pontos decimais.

Para uma melhor compreensão dessas variações, as Figuras 11 e 12 ilustram graficamente os comportamentos desses descritores.

Tabela 4: Dimensões fractais, $D_{f}$, parâmetros das regressões lineares e razões $\mathbf{r}_{f} / \mathbf{r}_{\text {}}$.

\begin{tabular}{|c|c|c|c|c|c|c|c|}
\hline BACIA & ESTADO & $\begin{array}{l}\text { Df- DIMENSÃO } \\
\text { FRACTAL }\end{array}$ & $r^{2}$ & $\varepsilon$ padr. & $\begin{array}{l}\text { COEFICIENTE } \\
\text { ANGULAR - } \beta_{1}\end{array}$ & $\begin{array}{l}\text { COEFICIENTE } \\
\text { LINEAR - } \beta O\end{array}$ & $\mathrm{rf} / \mathrm{ri}$ \\
\hline RIO CAPIBARIBE & PE & 1.1049545019 & 0.9505 & 0.0086 & -0.1049545019 & 7.1052985623 & 3.69 \\
\hline RIO UNA & PE & 1.1023034868 & 0.9511 & 0.0083 & -0.1023034868 & 6.9708264476 & 3.62 \\
\hline RIOSÃO BARTOLOMEU & GO-DF & 1.0972965771 & 0.9566 & 0.0083 & -0.0972965771 & 6.8741177733 & 3.86 \\
\hline RIO IPOJUCA & PE & 1.1100165304 & 0.9719 & 0.0068 & -0.1100165304 & 7.3009723881 & 3.67 \\
\hline RIO GOIANA & PE & 1.0620124944 & 0.9545 & 0.0052 & -0.0620124944 & 6.0321390146 & 3.95 \\
\hline RIOSINHAÉM & PE & 1.1391477291 & 0.9370 & 0.0132 & -0.1391477291 & 6.6557251516 & 3.68 \\
\hline RIOS JABOATÃO-PIRAPAMA & PE & 1.0957011193 & 0.9432 & 0.0084 & -0.0957011193 & 5.8475305872 & 3.60 \\
\hline BOTAFOGO_IGARASSÚ & PE & 1.1037531893 & 0.9122 & 0.0112 & -0.103753189 & 5.959816228 & 3.63 \\
\hline RIO DO AÇUDE DA CACHOEIRA II & PE & 1.0680547926 & 0.8895 & 0.0086 & -0.0680547926 & 5.1458634319 & 3.89 \\
\hline RIO SÃO JOÃO & MG & 1.0719445522 & 0.9374 & 0.0069 & -0.0719445522 & 4.7688169555 & 3.92 \\
\hline RIO SÃO JOÃO - A & PR & 1.0756555150 & 0.9394 & 0.0068 & -0.0756555150 & 4.6403414775 & 3.83 \\
\hline RIOS ARIMBII-TAPERA-MEREPÊ & PE & 1.0730646409 & 0.9420 & 0.0066 & -0.0730646409 & 4.5977422897 & 3.67 \\
\hline R. DA CACHOEIRA DAS POMBAS & MG & 1.0360852426 & 0.8778 & 0.0041 & -0.0360852426 & 2.6158564886 & 3.03 \\
\hline
\end{tabular}


Tabela 5: Idem, sub-bacias da bacia do Riacho da Cachoeira das Pombas.

\begin{tabular}{ccccccc}
\hline $\begin{array}{c}\text { R. DA CACHOEIRA DAS } \\
\text { POMBAS }\end{array}$ & $\begin{array}{c}\text { Df- DIMENSÃO } \\
\text { FRACTAL }\end{array}$ & $r^{2}$ & $\varepsilon$ padr. & $\begin{array}{c}\text { COEFICIENTE } \\
\text { ANGULAR }-\beta 1\end{array}$ & $\begin{array}{c}\text { COEFICIENTE } \\
\text { LINEAR - } \beta \text { ० }\end{array}$ & rf/ri \\
\hline SUB-BACIA 01 & $\mathbf{1 . 0 3 1 4 5 3 1 0 4 8}$ & 0.6165 & 0.0075 & -0.0314531048 & 1.7788844840 & $\mathbf{3 . 7 3}$ \\
SUB-BACIA 02 & $\mathbf{1 . 0 2 5 1 6 1 6 1 5 2}$ & 0.7457 & 0.0059 & -0.0251616152 & 1.6567295516 & $\mathbf{3 . 6 4}$ \\
SUB-BACIA 03 & $\mathbf{1 . 0 2 9 8 7 5 1 7 0 7}$ & 0.5755 & 0.0056 & -0.0298751707 & 1.7830147981 & $\mathbf{2 . 0 1}$ \\
SUB-BACIA 04 & $\mathbf{1 . 0 3 8 2 9 4 6 4 3 0}$ & 0.3827 & 0.0113 & -0.0382946430 & 1.3741144126 & $\mathbf{1 . 9 2}$ \\
SUB-BACIA 05 & $\mathbf{1 . 0 1 4 6 1 9 6 7 6 5}$ & 0.2189 & 0.0052 & -0.0146196765 & 1.3541257928 & $\mathbf{1 . 8 1}$ \\
INTERBACIA & $\mathbf{1 . 0 6 1 3 9 1 3 7 5 2}$ & 0.7566 & 0.0128 & -0.0613913752 & 2.4021763618 & $\mathbf{2 . 9 6}$ \\
\hline
\end{tabular}

Tabela 6: Características geométricas e dos descritores de compacidade das bacias estudadas.

\begin{tabular}{|c|c|c|c|c|c|c|c|c|c|c|}
\hline BACIA & NPC & $\begin{array}{c}\triangle \mathrm{NPC} \\
(\mathrm{S})\end{array}$ & $\frac{\text { ÁREA }}{\left(\mathrm{km}^{2}\right)}$ & $\begin{array}{l}\Delta \mathrm{A} \\
(\mathrm{S})\end{array}$ & $\begin{array}{c}\text { PERÍMETRO } \\
(\mathrm{km})\end{array}$ & $\begin{array}{l}\Delta P \\
(s)\end{array}$ & $\begin{array}{l}\text { kc- íNDICE DE } \\
\text { COMPACIDADE }\end{array}$ & $\begin{array}{l}\Delta \mathrm{kc} \\
(\mathrm{s})\end{array}$ & $\begin{array}{l}\text { RE- RAZÃO DE } \\
\text { CIRCULARIDADE }\end{array}$ & $\begin{array}{l}\Delta R C \\
(\mathrm{~S})\end{array}$ \\
\hline RIO CAPIBARIBE & 549 & \multirow{2}{*}{-56.83} & 7377.689 & \multirow{2}{*}{-0.02} & 614.349 & \multirow{2}{*}{-12.09} & 2.018 & \multirow{2}{*}{-12.08} & 0.246 & \multirow{2}{*}{ 29.37 } \\
\hline Idem, Regularizada & 237 & & 7375.925 & & 540.061 & & 1.774 & & 0.318 & \\
\hline RIO SÃO BARTOLOMEU & 278 & \multirow{2}{*}{-53.96} & 5537.125 & \multirow{2}{*}{-0.14} & 493.759 & \multirow{2}{*}{-12.43} & 1.872 & \multirow{2}{*}{-12.37} & 0.285 & \multirow{2}{*}{30.23} \\
\hline Idem, Regularizada & 128 & & 5529.193 & & 432.362 & & 1.640 & & 0.372 & \\
\hline RIO UNA & 659 & \multirow{2}{*}{-57.06} & 6740.166 & \multirow{2}{*}{-0.01} & 579.272 & \multirow{2}{*}{-13.70} & 1.990 & \multirow{2}{*}{-13.69} & 0.252 & \multirow{2}{*}{34.25} \\
\hline Idem, Regularizada & 283 & & 6739.575 & & 499.924 & & 1.718 & & 0.339 & \\
\hline RIO IPOJUCA & 842 & \multirow{2}{*}{-58.19} & 3453.371 & \multirow{2}{*}{0.05} & 755.728 & \multirow{2}{*}{-13.91} & 3.628 & \multirow{2}{*}{-13.93} & 0.076 & \\
\hline Idem, Regularizada & 352 & & 3455.051 & & 650.584 & & 3.122 & & 0.103 & 35.00 \\
\hline RIO GOIANA & 364 & & 2879.616 & & 289.759 & & 1.523 & & 0.431 & 19.91 \\
\hline Idem, Regularizada & 159 & -56.32 & 2877.095 & -0.09 & 264.499 & -8.72 & 1.391 & -8.68 & 0.517 & 19.91 \\
\hline RIO SINHAÉM & 383 & 56.40 & 2088.742 & 0.01 & 327.858 & & 2.024 & & 0.244 & 44.18 \\
\hline Idem, Regularizada & 167 & -56.40 & 2088.978 & 0.01 & 273.063 & -16.71 & 1.685 & -16.72 & 0.352 & 44.18 \\
\hline RIOS JABOATÃO-PIRAPAMA & 526 & 58.751 & 1255.372 & & 202.538 & & 1.613 & & 0.385 & 2470 \\
\hline Idem, Regularizada & 217 & -58.75 & 1254.993 & -0.03 & 181.346 & -10.46 & 1.444 & -10.45 & 0.480 & 24.70 \\
\hline RIOS BOTAFOGO-IGARASSÚ & 392 & & 1063.498 & & 214.500 & & 1.855 & & 0.290 & \\
\hline Idem, Regularizada & 169 & $-56,89$ & 1063.342 & -0.01 & 187.530 & -12.57 & 1.622 & -12.57 & 0.380 & 30.81 \\
\hline RIO DO AÇUDE DACACHOEIRA II & 299 & & 394.558 & & 113.980 & & 1.619 & & 0.382 & \\
\hline Idem, Regularizada & 129 & -56.86 & 394.445 & -0.03 & 108.933 & -4.43 & 1.547 & -4.41 & 0.418 & 9.45 \\
\hline RIO SÃO JOÃO & 289 & & 182.183 & & 83.499 & & 1.745 & & 0.328 & \\
\hline Idem, Regularizada & 120 & -58.48 & 182.120 & -0.03 & 74.238 & -11.09 & 1.552 & -11.08 & 0.415 & 26.46 \\
\hline RIO SĀO JOĀO - A & 325 & - 20 & 145.956 & 0.17 & 69.437 & 720 & 1.621 & 700 & 0.380 & 16.11 \\
\hline Idem, Regularizada & 132 & $=39.38$ & 145.710 & & 64.385 & -7.28 & 1.505 & & 0.442 & 10.11 \\
\hline RIOS ARIMBI-TAPERA-MEREPẼ & 245 & & 128.888 & & 65.465 & & 1.627 & & 0.378 & \\
\hline Idem, Regularizada & 107 & -56.33 & 128.669 & -0.17 & 62.537 & -4.47 & 1.555 & -4.39 & 0.413 & 9.40 \\
\hline R. DACACHOEIRA DAS POMBAS & 179 & 55.31 & 7.009 & -0.18 & 12.666 & & 1.350 & 10.05 & 0.549 & 23.59 \\
\hline Idem, Regularizada & 80 & ${ }^{-55.31}$ & 6.997 & -0.18 & 11.383 & -10.13 & 1.214 & -10.05 & 0.679 & 23.59 \\
\hline MÉOIAS: & & -56.98 & & -0.06 & & -10.62 & & -10.59 & & 25.65 \\
\hline DESVIOS PADR.: & & 1.41 & & 0.07 & & 3.49 & & 3.51 & & 9.70 \\
\hline
\end{tabular}

Tabela 7: Idem, relativamente às sub-bacias da bacia do Riacho da Cachoeira das Pombas.

\begin{tabular}{|c|c|c|c|c|c|c|c|c|c|c|}
\hline R. DACACH. DAS POMBAS & NPC & $\begin{array}{c}\triangle N P C \\
(\$)\end{array}$ & $\begin{array}{l}\text { AREA } \\
\left(\mathrm{km}^{2}\right)\end{array}$ & $\begin{array}{l}\Delta A \\
(\$ 6)\end{array}$ & $\frac{\text { PERIMETRO }}{(\mathrm{km})}$ & $\begin{array}{l}\Delta P \\
(\$)\end{array}$ & $\begin{array}{l}\mathrm{kc} \text { - } \text { INDICE DE } \\
\text { COMPACIDADE }\end{array}$ & $\begin{array}{l}\Delta \mathrm{kc} \\
(\mathrm{s})\end{array}$ & $\begin{array}{l}\text { RC-RAZÃO DE } \\
\text { CIRCULARIDADE }\end{array}$ & $\begin{array}{l}\Delta R c \\
{[\times] 1}\end{array}$ \\
\hline $\begin{array}{c}\text { SUB-BACIA 1 } \\
\text { Idem, Regularizada }\end{array}$ & $\begin{array}{l}105 \\
47\end{array}$ & -55.24 & $\begin{array}{l}1.269 \\
1.256\end{array}$ & -0.97 & $\begin{array}{l}5.642 \\
5.075\end{array}$ & -10.05 & $\begin{array}{l}1.413 \\
1.277\end{array}$ & -9.61 & $\begin{array}{l}0.501 \\
0.613\end{array}$ & 22,40 \\
\hline $\begin{array}{c}\text { SUB-BACIA 2 } \\
\text { Idem, Regularizada }\end{array}$ & $\begin{array}{l}86 \\
40\end{array}$ & -53.49 & $\begin{array}{l}1.248 \\
1.238\end{array}$ & -0.82 & $\begin{array}{l}5.163 \\
4.617\end{array}$ & -10.58 & $\begin{array}{l}1.304 \\
1.171\end{array}$ & -10.21 & $\begin{array}{l}0.588 \\
0.730\end{array}$ & 24.03 \\
\hline $\begin{array}{c}\text { SUB-BACIA 3 } \\
\text { Idem, Regularizada }\end{array}$ & $\begin{array}{l}91 \\
52 \\
\end{array}$ & -42.86 & $\begin{array}{l}1.511 \\
1.511 \\
\end{array}$ & -0.01 & $\begin{array}{l}5.655 \\
5.255 \\
\end{array}$ & -7.08 & $\begin{array}{l}1.298 \\
1.206 \\
\end{array}$ & -7.07 & $\begin{array}{l}0.594 \\
0.688\end{array}$ & 15.80 \\
\hline $\begin{array}{c}\text { SUB-BACIA 4 } \\
\text { Idem, Regularizada }\end{array}$ & $\begin{array}{l}69 \\
40 \\
\end{array}$ & -42.03 & $\begin{array}{l}0.684 \\
0.682\end{array}$ & -0.34 & $\begin{array}{l}3.661 \\
3.392 \\
\end{array}$ & -7.34 & $\begin{array}{l}1.249 \\
1.159\end{array}$ & -7.19 & $\begin{array}{l}0.641 \\
0.744\end{array}$ & 16.08 \\
\hline $\begin{array}{c}\text { SUB-BACIA 5 } \\
\text { Idem, Regularizada }\end{array}$ & $\begin{array}{l}68 \\
40\end{array}$ & -41.18 & $\begin{array}{l}0.786 \\
0.781 \\
\end{array}$ & -0.61 & $\begin{array}{l}3.916 \\
3.622 \\
\end{array}$ & -7.49 & $\begin{array}{l}1.246 \\
1.156 \\
\end{array}$ & -7.21 & $\begin{array}{l}0.644 \\
0.748 \\
\end{array}$ & 16.13 \\
\hline $\begin{array}{c}\text { INTERBACIA } \\
\text { Idem, Regularizada }\end{array}$ & $\begin{array}{r}164 \\
75\end{array}$ & -54.27 & $\begin{array}{l}1.519 \\
1.506\end{array}$ & -0.85 & $\begin{array}{l}9.352 \\
8.434\end{array}$ & -9.82 & $\begin{array}{l}2.141 \\
1.939\end{array}$ & -9.43 & $\begin{array}{l}0.218 \\
0.266\end{array}$ & 21.92 \\
\hline $\begin{array}{l}\text { MÉDIAS: } \\
\text { DESVIOS PADR.: }\end{array}$ & & $\begin{array}{c}-48.18 \\
6.20\end{array}$ & & $\begin{array}{l}-0.60 \\
0.33\end{array}$ & & $\begin{array}{l}-8.73 \\
1.45\end{array}$ & & $\begin{array}{r}-8.45 \\
1.32\end{array}$ & & $\begin{array}{r}19.40 \\
3.45\end{array}$ \\
\hline
\end{tabular}




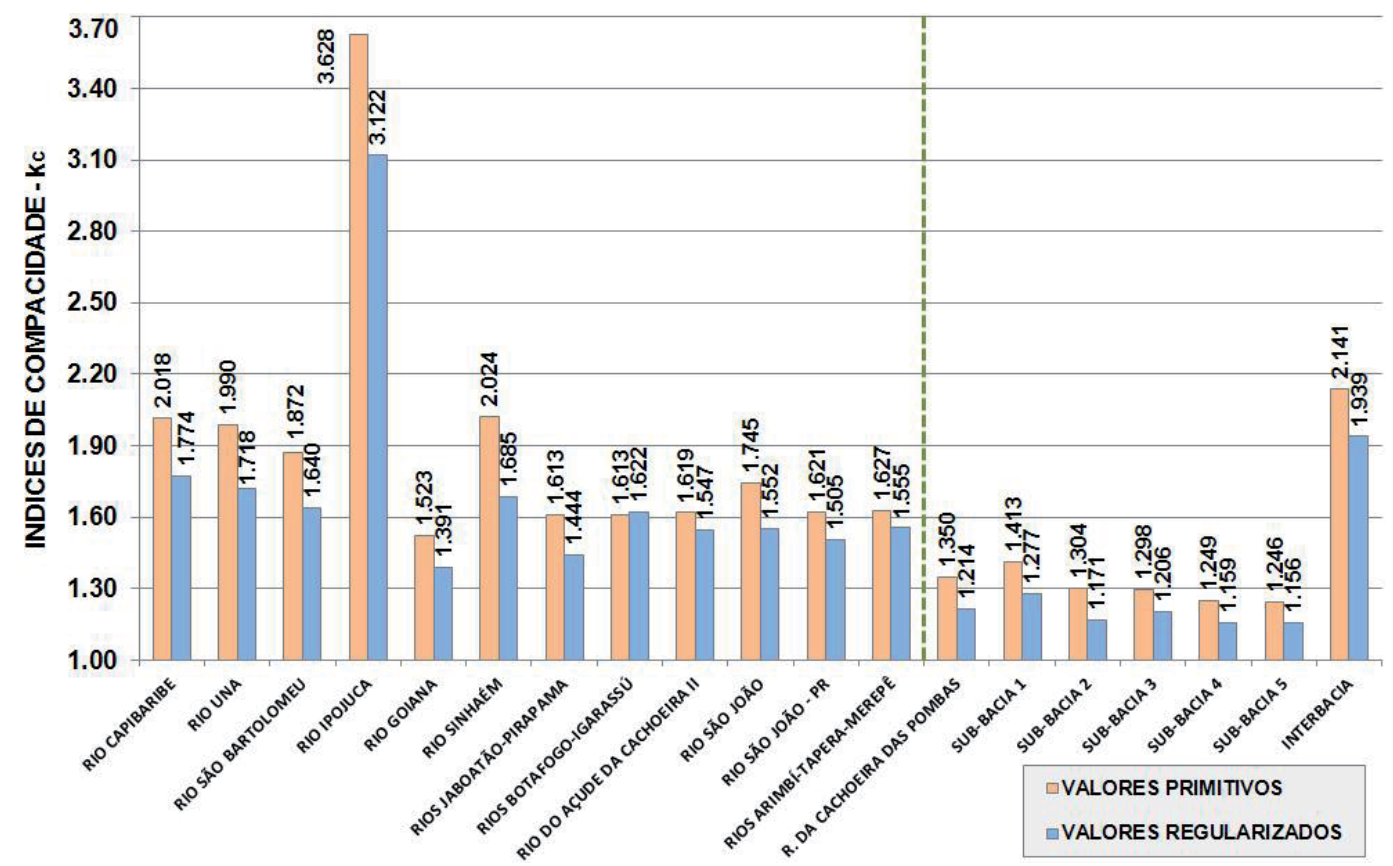

Figura 11 - Índices de Compacidade, $K_{C}$, calculados com os perímetros primitivo e regularizado.

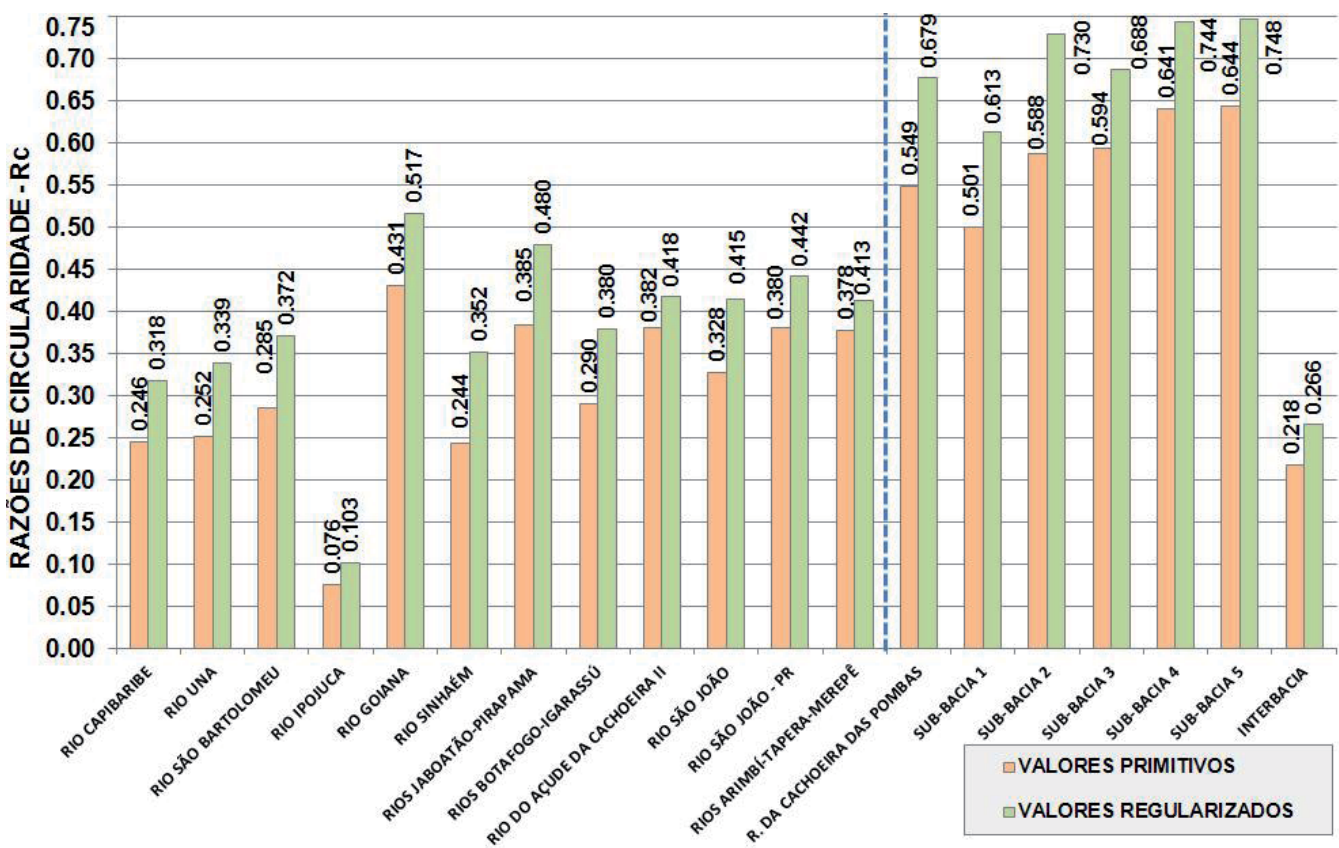

Figura 12 - Razões de Circularidade, $R_{C}$, calculadas com os perímetros primitivo e regularizado.

\section{Análise dos Resultados}

Da análise das informações apresentadas na Tabela 4, depreende-se a boa qualidade das regressões lineares procedidas, em especial relativamente aos erros absolutos computados. Esse fato garante, por consequência, a qualidade das dimensões fractais, $\mathrm{D}_{\mathrm{f}}$, determinadas para as diferentes bacias estudadas.
Por outro lado, depreende-se que as razões entre as escalas finais e iniciais adotadas nos procedimentos de caminhamento, $r_{f} / r_{i}$, não atingiram o número adequado e igual a 4, proposto por Bazănt, ocorrência que também se observa, entretanto mais claramente, nos resultados apresentados na Tabela 5. Esse fato decorre especialmente da limitação inferior imposta ao número 
de pontos no contorno, da ordem de $12 \%$ (a qual não deve ser relaxada), situação que pode ser contornada aumentando-se a discretização geométrica do contorno primitivo, sobretudo para bacias menores e sub-bacias.

Porém, a faixa de admissibilidade proposta neste artigo, a qual situa $r_{f} / r_{i}$ entre 3 e 4 , foi satisfatoriamente atingida. Como referido na premissa de número 5 do Capítulo 4, entende-se que razões variando dentro dessa faixa sejam amplas o suficiente para o cômputo da dimensão fractal. Com efeito, as diferenças verificadas de $r_{f} / r_{i}$ relativamente a 4 , passam a refletir numericamente sobre os valores de $\mathrm{D}_{\mathrm{f}}$, pós a quinta casa decimal.

Considera-se, complementarmente, que $\mathrm{D}_{\mathrm{f}}$, no presente caso, constitui-se exclusivamente em um indicador da rugosidade associada ao contorno da forma. Considerando-se que o objetivo principal deste trabalho focou a determinação de contornos e perímetros "regularizados" que conduzam ao aumento da qualidade dos descritores de compacidade, o fato em discussão é relevante, unicamente no que diz respeito à aplicabilidade das Equações numeradas de 20 a 23.

Por outro lado, ao analisarem-se as informações contidas nas Tabelas 6 e 7, observa-se a drástica redução do número de pontos nos contornos regularizados, comparativamente àqueles relativos aos contornos primitivos (da ordem de $57 \%$ para as bacias de maiores dimensões e de $48 \%$ para as sub-bacias analisadas). Entretanto, as variações das áreas mostraram-se insignificantes.

Da mesma maneira, as variações médias, a menor, dos Índices de Compacidade, $\mathrm{k}_{\mathrm{C}}$ foram da ordem de 11\% para as bacias maiores e de $8 \%$ para as sub-bacias. Da maneira similar, as variações das Razões de Circularidade, $\mathrm{R}_{\mathrm{C}}$, apresentaram valores médios, a maior, da ordem de $26 \%$ e $19 \%$, para bacias maiores e sub-bacias, respectivamente, fatos relevantes que ficam evidenciados nas Tabelas 6 e 7 (gráficos das Figuras 11 e 12).

\section{Conclusões}

Neste trabalho, um novo modelo fundamentado na análise fractal foi proposto para a determinação de contornos regularizados e de perímetros a eles associados, objetivando a determinação de valores consistentes e mais realistas, de descritores morfométricos de compacidade associados às bacias hidrográficas.

Em virtude dos fatos noticiados ao longo do texto
(Capítulo 5), conclui-se que o Modelo de Caminhamento sobre o Contorno pode ser utilizado com eficácia e segurança para a análise de formas legitimamente fractais (autossimilares ou autoafins), desde que medidas restritivas como aquelas aqui propostas sejam observadas, o que se aplica às bacias hidrográficas, não obstante se desconheçam valores teóricos de $\mathrm{D}_{\mathrm{f}}$ para contornos aleatórios.

A partir dos resultados apresentados e das análises a eles relacionadas, ressaltam-se, conclusivamente, as reduções verificadas nos Índices de Compacidade, $\mathrm{k}_{\mathrm{C}}$, com consequentes aumentos das Razões de Circularidade, $\mathrm{R}_{\mathrm{C}}$, parâmetros fundamentais para o desenvolvimento de análises morfométricas, especialmente aquelas afetas à avaliação de riscos de natureza hidrológica.

Verifica-se, ainda, a preservação das formas primitivas das bacias conforme se depreende da análise da Figura 9B. Enfatiza-se, finalmente, que os parâmetros de compacidade calculados da maneira clássica, parecem subavaliar as reais características de compacidade e de circularidade das bacias, o que pode conduzir a equívocos, relativamente à avaliação dos tempos de concentração dos caudais, assim como dos níveis de severidade de enchentes e picos de cheia, fato que se tem observado com certa frequência nos dias atuais.

\section{Agradecimentos}

O presente trabalho não foi suportado por agências de fomento. Ainda, não teve origens em projetos de pesquisas financiados por universidades ou outras organizações.

Entretanto, os autores manifestam os seus agradecimentos à FAPESP - Fundação de Amparo à Pesquisa do Estado de São Paulo, ao CNPq- Conselho Nacional de Desenvolvimento Científico e Tecnológico e a CAPES- Coordenação de Aperfeiçoamento de Pessoal de Nível Superior, organismos brasileiros de fomento à pesquisa, pelo suporte continuado disponibilizado aos pesquisadores nas diversas áreas do conhecimento.

\section{Referências Bibliográficas}

BAZǍNT, Z. P. Size effect in blunt fracture: concrete, rock, metal. Journal of Engineering Mechanics, ASCE, 110, p. 518-535,1984.

BAZǍNT, Z. P.; PFEIFFER, P. A. Determination of Fracture 
Energy from Size Effect and Brittleness Number. ACI Materials Journal, Title 84M41, p. 463-480, Nov./Dec., 1987.

BAZǍNT, Z. P.; KAZEMI, M. T. Determination of fracture energy, process zone length and brittleness number from size effect, with applications to rock and concrete. International Journal of Fracture, v. 44, n. 2, p. 111-131, 1990.

BLACHOWSKI, A.; RUEBENBAUER, K. Roughness Method to Estimate Fractal Dimension. Acta Physica Polonica A, v. 115, n. 3, p. 636-640, 2009.

CLARK, N. Three techniques for implementing digital fractal analysis of particle shape. Powder Technology, v. 46, n. 1, p. 45-52, 1986.

CLARKE, K.; SCHWEIZER, D. Measuring the Fractal Dimension of Natural Surfaces Using a Robust Fractal Estimator. Cartography and Geographic Information and Systems, v. 18, n. 1, p. 37-47, 1991 .

CONSTANTIN, L. V.; IORDACHE, D. A. Study of the Fractal and Multifractal Scaling Intervening in the Description of Fracture Experimental Data Reported by the Classical Work: Nature 308, 721-722 (1984). Mathematical Problems in Engineering, article ID706326, DOI: 10.1155/2012/706326, 2012.

DEEPA, S.; TESSAMMA, T. Fractal Features based on Differential Box Counting Method for the Categorization of Digital Mammograms. International Journal of Computer Information System and Industrial Management Applications, v. 2, p. 11-19, 2010.

FALCONER, K. J. Fractal Geometry - Mathematical Foundations and Applications. John Wiley \& Sons, 1990.

FERREIRA, C. W. S.; LIMA, C. S.; CAVALCANTI, L. C. S.; SANTOS, A. O. Caracterização Morfométrica da Bacia Hidrográfica do Açude Cachoeira II. In: VI Seminário Latino Americano de Geografia Física e II Seminário Ibero Americano de Geografia Física. Anais...Universidade de Coimbra, p. 1-10, 2010.

FERREIRA, L. E. T. FloRiSys -Um sistema computacional destinado à análise de riscos hidrometeorológico (disponível para uso acadêmico mediante contato com autor: luizeduardotferreira@ gmail.com), 2014

GOODCHILD, M. F. Fractals and the Accuracy of Geographical Measures. Mathematical Geology, v. 12, n. 2, p. 85-98, 1980.

GRAVELIUS, H. Flusshunde. In: Goschen'sche (Ed.), Berlin (AL): Verlagshabd-lung, 1914
HUANG, Z. H.; TIAN, J. F.; WANG, Z. G. Study of the slit island analysis as a method for measuring fractal dimension of fractured surface. Scripta Metallurgica et Materialia, v. 24, n. 6, p. 962-972, 1990.

ISSA, MOHSEN A.; ISSA, MAHMOUD A.; ISLAM, MD. S., CHUDNOVSKY A. Fractal dimension - a measure of fracture roughness and toughness of concrete. Engineering Fracture Mechanics, v. 70, n. 1, p. 125-137, 2003.

JULCA, S. B. M. Magnitud, frecuencia y factores que controlan los flujos sedimentarios desde los andes centrales occidentales hacia el océano pacífico peruano. Tese (Doutorado). Lima (Perú): Universidad Nacional Agraria La Molina, 2014.

KLIKEMBERG, B. A rewiew of Methods Used to Determine the Fractal Dimension of Linear Features. Mathematical Geology, v. 26, n.1, p. 23-46, 1994.

LANCASTRE, A.; FRANCO, F. M. Lições de Hidrologia. Monte da Taparica (PT): Universidade Nova Lisboa, 1984.

LAUX, P. R.; PEREIRA, R. S. Revisão do Modelo de Geometria Fractal. XII Simpósio Brasileiro de Sensoriamento Remoto Anais, Goiânia (BR): INPE, p. 14103-4110, 2005.

LORENZ, E. N. The Essence of Chaos. Seattle (USA): University of Washington Press, 1995.

LOVEJOY, S. Area - perimeter relation for rain and cloud areas. Science, v. 216, n. 9, 1982.

LUNG, C. W.; MU, Z. Q. Fractal dimension measured with perimeter-area relation and toughness of materials. Physical Review B, v. 38, n. 16, p. 11781-11784, 1988.

MANDELBROT, B. B. How long is the coast of Britain? Statistical self-similarity and fractional dimension. Science, v. 156, p. 636-638, 1967.

MANDELBROT, B. B. Fractals: Form, Chance and Dimension. San Francisco, (USA): Freeman, 1977.

MANDELBROT, B. B. Objectos Fractais. Lisboa (PT): Gradiva, 1991.

MECHOLSKY, J. J.; PASSOJA, D. E.; FEINBERG-RINGEL, K. S. Quantitative analysis of brittle fracture surfaces using fractal geometry, Journal of the American Ceramics Society, v. 72, n. $1,1989$.

MILLER, V. C. A quantitative geomorphic study of drainage basin characteristics in the Clinch mountain area, Virginia and Tennessee. Office of Naval Research, Geography Branch. Project NR 389-042, Technical Report 3, Columbia University, 
USA, 1953.

PANDE, C. S.; RICHARDS, L. E. ; LOUAT, N.; DEMPSEY, V.; SCHWOEBLE, A. J. Fractal characterization of fractured surfaces. Acta Metallurgica, v. 35, n. 7, p. 1633-1637, 1987.

RAMANUJAN, S. Ramanujan's Collected Works, New York (USA): Chelsea, 1962.

RICHARDSON, L. F. Fractal growth phenomena. Ann Arbor, Michigan (USA): The Society, 6, 1961.

RILEM- Technical Committee- 89 - FMT- Size Effect Method for Determining Fracture Energy and Process Zone Size of Concrete. - Draft Recommendations. Materials and Structures, 23, p. 461-465, 1990.

SALLES, M. M. O Uso do SIG na Análise Morfométrica da Bacia Hidrográfica do Rio São João - MG. Instituto de Geociências, Departamento de Cartografia da Universidade Federal de Minas Gerais, UFMG, Monografia (Especialização), 2010.

SANTOS, E.; BARRETO, K.; ROCHA, C.; WEIRICH NETO, P. Uso das Terras na Bacia do Rio São João, Carambeí-PR. 10 Conex - Encontro Conversando sobre Extensão. Pró-Reitoria de Extensão e Assuntos Culturais, Universidade Estadual de Ponta Grossa (UEPG), 2012.

SARKER, N.; CHAUDHURI, B. B. An efficient differential box-counting approach to compute fractal dimension of image. IEEE Transaction on Systems, Man, and Cybernetics, v.24, n. 1, p. 115-120, 1994.

SCHEIDEGGER, A. E. Theoretical Geomorphology. 2nd ed., New York (USA): Springer Verlag, 1970.

SHELBERG, M. C.; MOELLERING, H.; LAM, N. Measuring the fractal dimensions of empirical cartographic curves. Defense Mapping Agency-Aerospace Center/CDCT, ASIN: B00072MFUY, p. 481-490, 1982.

SENA-SOUZA, J.P.; MARTINS, E. S.; COUTO JÚNIOR, A. F.; REATTO, A.; VASCONCELOS, V.; GOMES, M. P.; CARVALHO JÚNIOR, O. A.; REIS, A. M. Mapeamento Geomorfológico da Bacia Hidrográfica do Rio São Bartolomeu, escala 1:100.000. Boletim de Pesquisa e Desenvolvimento 314, ISSN on line 2176-509X, Planaltina (DF): EMBRAPA Cerrados, 2013.

TONELlO, K. C. Análise Hidroambiental da Bacia Hidrográfica da Cachoeira das Pombas, Guanhães, MG. Dissertação (Mestrado), Viçosa (MG): Universidade Federal de Viçosa. 2005.

UNDERWOOD, E. E.; BANERJI, K. Fractals in fractography, Materials Science and Engineering, v. 80, n. 1, p. 1-14, 1986.

VILLELA, S. M.; MATTOS, A. Hidrologia Aplicada. São Paulo (SP): Editora McGraw-Hill, 1975. 